\title{
How realistic are the wakes of scaled wind turbine models?
}

\author{
Chengyu Wang ${ }^{1}$, Filippo Campagnolo ${ }^{1}$, Helena Canet $^{1}$, Daniel J. Barreiro ${ }^{1}$, and Carlo L. Bottasso ${ }^{1}$ \\ ${ }^{1}$ Wind Energy Institute, Technische Universität München, D-85748 Garching b. München, Germany
}

Correspondence: Carlo L. Bottasso (carlo.bottasso@tum.de)

\begin{abstract}
.
The aim of this paper is to analyze to which extent wind tunnel experiments can represent the behavior of full-scale wind turbine wakes. The question is relevant because on the one hand scaled models are extensively used for wake and farm control studies, whereas on the other hand not all wake-relevant physical characteristics of a full-scale turbine can be exactly matched by a scaled model. In particular, a detailed scaling analysis reveals that the scaled model accurately represents the principal physical phenomena taking place in the outer shell of the near wake, whereas differences exist in its inner core. A large eddy simulation actuator line method is first validated with respect to wind tunnel measurements, and then used to perform a detailed comparison of the wake at the two scales. It is concluded that, notwithstanding the existence of some mismatched effects, the scaled wake is remarkably similar to the full-scale one, except in the immediate proximity of the rotor.
\end{abstract}

\section{Introduction}

The simulation of wind turbine wakes in wind tunnels has been gaining an increasing interest in recent years. In fact, since wakes represent a major form of coupling within a wind plant, understanding their behavior and accurately simulating their effects are today problems of central importance in wind energy science, with direct practical implications on design, operation and maintenance. Recent studies include the analysis of single and multiple interacting wakes (see, for example, the review in Bottasso and Campagnolo (2020) or, among others, Whale et al. (1996); Chamorro and Porté-Agel (2009, 2010); Bartl and Sætran (2016); Bastankhah and Porté-Agel (2016); Tian et al. (2018); Campagnolo et al. (2016); Bottasso et al. (2014a); Campagnolo et al. (2020); Wang et al. (2020c) and references therein).

Wind tunnel testing offers some unique advantages over full-scale field testing:

- The ambient conditions are repeatable and —at least to some extent— controllable.

- Detailed flow measurements are possible with a plethora of devices, from standard pressure and hot-wire probes, to PIV (Meinhart, 1999) and scanning lidars (van Dooren et al., 2017), whereas measurements of comparable accuracy are today hardly possible at full scale. Additionally, time flows faster in a scaled experiment than at full scale (Bottasso and Campagnolo, 2020; Canet et al., 2020; Campagnolo et al., 2020), which means that a large informational content can be accumulated over relatively short periods of time. 
- Models can be designed ad hoc to achieve specific goals, and can be extensively instrumented (Bottasso and Campagnolo, 2020), while layouts and scenarios can be readily changed to explore different operating conditions of interest.

- Costs are limited, even for highly sophisticated models, also because there are no energy production losses as it is often the case in the field, whereas the costs of sophisticated wind tunnel facilities are typically amortized by their use for several different applications over long periods of time.

- Open datasets can be shared within the research community and collaborations are facilitated, since there are no —or fewer- constraints from intellectual property than when real wind turbine data is used.

Testing in the controlled and repeatable environment of the wind tunnel is today contributing to the understanding of the physical processes at play, generates valuable data for the validation and calibration of mathematical models, and offers opportunities for the verification of control technologies.

However, notwithstanding these and other unique advantages, a major question still hovers over the wind tunnel simulation of wakes: how faithful are these wakes to the actual ones in the field? In fact, in private conversations these authors have often been questioned on the actual usefulness of wind tunnel testing, based on a perceived lack of realism of these scaled tests. Indeed, some skepticism is justified and completely understandable: simulation codes are being calibrated and validated with respect to wind tunnel measurements, and wind farm control techniques are being compared and evaluated in wind tunnel experiments. Therefore, it is important to quantify the level of realism of wind tunnel simulated wakes, and to identify with better clarity what aspects faithfully represent the full-scale truth and what aspects do not.

A thorough and complete answer to this question is probably still out of reach today. In fact, detailed inflow and wake measurements of a full-scale turbine would be necessary, with a level of detail comparable to the ones achievable in the tunnel. Lidar technology is making great progress (Zhan, 2020), and might soon deliver suitable datasets. It should be a goal of the scientific and industrial communities to completely open such future datasets to research, which would surely greatly favor the scientific advancement of the field. In the meanwhile, however, some partial answers to the question of wake realism can still be given. This is the main goal of the present paper.

This study considers the TUM G1 scaled wind turbine (Bottasso and Campagnolo, 2020), and a dataset obtained with this machine in the boundary layer wind tunnel of the Politecnico di Milano in Italy. A large eddy simulation (LES) actuator line method (ALM) (Wang et al., 2019) is used to simulate the wind tunnel experiments, including the passive generation of a sheared turbulent inflow. The code has been validated with respect to the present and other similar measurements.

Following Bottasso and Campagnolo (2020) and Canet et al. (2020), dimensional analysis and wake physics are used to review the main factors driving wake behavior. The same analysis also reveals which physical aspects of full-scale wakes cannot be matched at the reduced scale and with the considered experimental setup. A first analysis of scaling was performed by Chamorro et al. (2016), considering the effects caused by the mismatch of the rotor-based Reynolds. Experimental results based on a miniature wind turbine showed that wake behaviour is unaffected by this parameter when it is larger than circa $10^{5}$. However, in reality the behavior of the blades and, as a consequence, of the wake is much more strongly affected by the chord-based Reynolds number, as initially discussed in Bottasso et al. (2014a). In fact, the much lower Reynolds regime of a 
small-scale model blade compared to a full-scale machine implies very different aerodynamic characteristics of the airfoils, which in turn drive a number of specific design choices of the scaled model (Bottasso and Campagnolo, 2020; Canet et al., 2020). Notwithstanding the differences caused by the chord-based Reynolds mismatch, it is relatively easy —as shown more in detail later on - to match the main processes taking place in the outer shell of the near wake, as well as the ones that govern its breakdown and the characteristics of the far wake. On the other hand, several mismatched effects do exist in the central core of the near wake. Dimensional analysis also expresses the scaling relationships that allow the mapping of scaled quantities into equivalent full-scale ones, and viceversa.

Based on the understanding provided by dimensional analysis and wake physics, full-scale turbines are designed in this work to match some of the G1 scaled-model parameters. Various versions of these models are considered, ranging from a more realistic full-scale turbine - with a larger number of mismatched effects - to less realistic ones that however match a larger set of quantities of the scaled model.

The full-scale models are then simulated with the LES-ALM code, using the same exact numerical methods and algorithmic parameters used for the scaled simulations. These wind turbine models are also exposed to the same identical ambient turbulent inflow used for the scaled model. The underlying assumption is that, since the code was found to be in very good agreement with measurements obtained in the scaled experiments, the same code based on the same numerical setup should deliver results of similar accuracy even at full scale. This assumption cannot be formally proven at this stage, but it seems to be very reasonable and it is probably the only possible approach that can be pursued in the absence of a detailed full-scale dataset.

Finally, the numerically simulated scaled and full-scale wakes are compared. The analysis considers wind-aligned and misaligned conditions, typical of wake steering control applications, and various metrics, including wake shape, path, speed profile, Reynolds shear stresses, power available and wind direction modification due to the curled wake in misaligned conditions. This detailed comparison is used to quantify the degree of similarity among the different models and across the various metrics. Since the models differ by known mismatched effects, this also helps pinpoint and explain any source of discrepancy.

The paper is organized according to the following plan. Section 2 uses dimensional analysis and wake physics to identify the quantities that can be exactly matched between scaled and full-scale models, the ones that can only be partially matched, the ones that are unmatched, and those that are neglected from the present analysis. Next, Section 3 describes the scaled experimental wind turbine and its full-scale counterparts, which include various modifications to highlight the effects of specific mismatches. Section 4 describes the numerical simulation model, including the generation of the turbulent inflow in the wind tunnel. Results and detailed comparisons among the scaled and the full-scale models are reported in Section 5. Finally, Section 6 summarizes the main findings of this work.

\section{Scaling}

The matched, partially matched, unmatched and neglected physical effects of the scaled and full-scale models are reviewed

next. Quantities referred to the scaled model are indicated with the subscript $(\cdot)_{M}$, while quantities referred to the full-scale physical system with the subscript $(\cdot)_{P}$. Scaling is defined by two parameters (Bottasso and Campagnolo, 2020; Canet et al., 
2020): the length scale factor $n_{l}=l_{M} / l_{P}$, where $l$ is a characteristic length (for example the rotor radius $R$ ), and the time compression ratio $n_{t}=t_{M} / t_{P}$, where $t$ is time. In the present case $n_{l}=1 / 162.1$ and $n_{t}=1 / 82.5$. A more complete treatment of scaling for wind turbine rotors is given in Bottasso and Campagnolo (2020) and Canet et al. (2020).

\subsection{Matched quantities}

- Inflow. The ambient flow is obtained by simulating the passive generation of turbulence in the wind tunnel, as explained in $\S 4.2$; the developed flow is sampled on a rectangular plane, which becomes the inflow of the scaled turbine simulations. For the full-scale turbine simulations, the sides of the inflow rectangular area are geometrically scaled by $n_{l}$, while time is scaled by $n_{t}$ and speed $V$ as $V_{M} / V_{P}=n_{l} / n_{t}$, resulting in a flow with exactly the same identical characteristics (e.g., shear, turbulence intensity, integral length scale, etc.) at the two scales.

- Tip speed ratio (TSR) $\lambda=\Omega R / V$, where $\Omega$ is the rotor speed. TSR determines not only the triangle of velocity at the blade sections, but also the pitch of the helical vortex filaments shed by the blade tips.

- Non-dimensional circulation $\Gamma(r) /(R V)=1 / 2(c(r) / R) C_{L}(r)(W(r) / V)$, where $C_{L}$ is the lift coefficient, $c$ the local chord, $W$ the local flow speed relative to the blade section, and $r$ is the spanwise blade coordinate (Burton et al., 2011). Each blade sheds trailing vorticity that is proportional to the spatial (spanwise) gradient $\mathrm{d} \Gamma / \mathrm{d} r$. Therefore, matching the non-dimensional spanwise distribution of $\Gamma$ (and, hence, also its non-dimensional spanwise gradient) ensures that the two rotors shed the same trailing vorticity.

The root of the G1 blade is located further away from the rotor axis than a typical full-scale machine, due to the space required for housing the pitch actuation system. The resulting effects caused on the wake were investigated by developing two different full-scale models, one with the exact same non-dimensional circulation of the G1 and one with more typical full-scale values, as discussed later.

- Rotor vortex shedding. The rotor Strouhal number $\mathrm{St}=f 2 R / V$ is matched, where $f$ is the rotor vortex-shedding characteristic frequency, which ensures the correct periodic release of vortices behind the rotor.

\subsection{Approximatively matched quantities}

The following quantities or effects are very nearly, but not exactly, matched:

- Thrust coefficient $C_{T}=T /\left(1 / 2 \rho A V^{2}\right)$, where $T$ is the thrust force, $\rho$ is air density and $A=\pi R^{2}$ the rotor swept area. The thrust characterizes to a large extent the speed deficit in the wake. In misaligned conditions, it is also the principal cause for the lateral deflection of the wake. The thrust coefficient is very nearly matched whereas the power coefficient is not (as discussed later), because the latter strongly depends on airfoil efficiency, which is affected by the Reynolds mismatch between the two models. On the other hand, drag has only a limited effect on thrust, which as a result is very similar in the two models. 
- Dynamic spanwise vortex shedding. During transients, spanwise vorticity is shed that is proportional to the temporal gradient of the circulation. To match the spanwise vortex shedding of a rotor, the matching of $(1 / R V) \mathrm{d} \Gamma / \mathrm{d} \tau$ should be ensured (Bottasso and Campagnolo, 2020; Canet et al., 2020), where $\tau$ is a non-dimensional time (for example, $\tau=\Omega_{r} t$, $\Omega_{r}$ being a reference rotor speed), equal for both the full and scaled models.

Rewriting the circulation as

$$
\frac{\Gamma}{R V}=\frac{1}{2} \frac{c}{R} C_{L_{\alpha}} \frac{W}{V}\left(\frac{U_{P} U_{T}}{W^{2}}-\theta\right)
$$

$C_{L_{\alpha}}$ being the lift curve slope, the dynamic spanwise vortex shedding condition implies the matching of the nondimensional time rates of change of the sectional tangential and perpendicular flow components $U_{P}$ and $U_{T}$, with $W^{2}=U_{P}^{2}+U_{T}^{2}$, and of the pitch angle $\theta$. The flow speed component tangential to the rotor disk is $U_{T}=\Omega r+u_{T}$, where $u_{T}$ contains terms due to wake swirl and yaw misalignment. The flow speed component perpendicular to the rotor disk is $U_{P}=(1-a) V+u_{P}$, where $a$ is the axial induction factor, and $u_{P}$ the contribution due to yaw misalignment and vertical shear. A correct similitude of dynamic vortex shedding is ensured if the non-dimensional time derivatives $\lambda^{\prime}, a^{\prime}$, $u_{P}^{\prime}, u_{T}^{\prime}$ and $\theta^{\prime}$ are matched, where $(\cdot)^{\prime}=\mathrm{d} \cdot / \mathrm{d} \tau$.

Matching of $\lambda^{\prime}$ is ensured here by the fact that the two rotors operate at the same TSR in the same inflow; additionally, the simulations were conducted by prescribing the rotor rotation (i.e. without a controller in the loop), so that $\Omega^{\prime}=0$. The term $a^{\prime}$ accounts for dynamic changes in the induction, which are due to the speed of actuation (of torque and blade pitch) and by the intrinsic dynamics of the wake. The speed of actuation is not relevant in this case, due to the absence of a pitch-torque controller. The intrinsic dynamics of the wake, as modelled by a first order differential equation (Pitt and Peters, 1981), is also automatically matched thanks to the matching of the TSR (Bottasso and Campagnolo, 2020; Canet et al., 2020). Finally, $u_{P}^{\prime}$ and $u_{T}^{\prime}$ are matched because the inflow is the same, with the exception of the contribution of wake swirl, which is not exactly the same because of the different torque coefficient, as noted below.

- Inflow size. The cross section of the wind tunnel has a limited size, resulting in the blockage phenomenon, i.e. in an acceleration of the flow between the object being tested and the sides (lateral walls and ceiling) of the tunnel (Chen and Liou, 2011). Although this problem is not strictly related to the scaling laws discussed here, it is still an effect that needs to be accounted for, especially if the ratio of the frontal area of the tested objected and the cross sectional area of the tunnel is not negligible. Simulations in domains of increasingly larger cross sections are conducted to quantify the blockage affecting the experimental setup considered here.

- Integral length scales (ILS). For the size of the TUM G1 turbines, the wind tunnel used in this research (located at Politecnico di Milano, Italy) generates a full-scale ILS of approximately $142 \mathrm{~m}$ at hub height, which is respectively about $16 \%$ and 58\% smaller that the lengths specified by Ed. 2 (IEC 61400-1, 1999) and Ed. 3 (IEC 61400-1, 2005) of the IEC 61400-1 international standards. To understand the effects of this mismatch on wake behavior, different simulations are conducted in turbulent inflows differing only in their integral scales. 


\subsection{Unmatched quantities}

The following quantities cannot be matched based on the current experimental setup and scaling choices:

- The chord-based Reynolds number $\operatorname{Re}=\rho W c / \mu$, where $\mu$ is the fluid viscosity. The Reynolds mismatch is $\operatorname{Re}_{M} / \operatorname{Re}_{P}=$ $n_{l}^{2} / n_{t}$, which is equal to 318.5 in the present case. This implies that the blades of the G1 model operate in a very different regime than the ones of the full-scale blade (Lissaman, 1983). To mitigate these effects, the G1 blade has a larger chord than the full-scale one, and uses ad hoc low-Reynolds airfoils (Bottasso and Campagnolo, 2020; Lyon and Selig, 1996). Additionally, noting that the scaling relationship of the rotor speed is $\Omega_{M} / \Omega_{P}=1 / n_{t}$, the time compression ratio $n_{t}$ was chosen to further increase Reynolds on the scaled blade and reduce its mismatch (Bottasso and Campagnolo, 2020).

- The power coefficient $C_{P}=P /\left(1 / 2 \rho A V^{3}\right)$, where $P$ is the aerodynamic power. The power coefficient of the scaled model is lower than the one of the full-scale machine, because of the smaller efficiency of the airfoils at low-Reynolds regimes. Since the torque coefficient is $C_{Q}=C_{P} / \lambda$, then also $C_{Q}$ is unmatched and lower for the small-scale model than for the full-scale one, resulting in reduced wake swirling (Burton et al., 2011).

- Tower and nacelle vortex shedding. The tower Strouhal number $\mathrm{St}=f d / V$ is matched when the tower diameter $d$ is geometrically scaled. However, as noted later, the diameter of the G1 tower is $49 \%$ larger than the one of the full-scale machine, so that frequency and size of the shed vortices is accordingly affected. An even larger mismatch applies to the nacelle, which has a frontal area that is 2.6 times larger in the scaled model.

- Stall delay due to rotational augmentation (Dowler and Schmitz, 2015). Matching these effects requires the matching of the blade chord and twist distributions, of the non-dimensional circulation and of the Rossby number Ro $=\Omega r /(2 W)$ (Bottasso and Campagnolo, 2020). While the latter two quantities are indeed matched, the former two are not to compensate for Reynolds mismatch. The G1 simulations were conducted without correcting the inboard airfoils for rotational augmentation. To quantify the effects of rotational augmentation on wake behavior, two versions of the full-scale turbine were developed, as explained later on.

- The chord-based Mach number Ma $=W / s$, where $s$ is the speed of sound. However compressibility effects are irrelevant for the full and scaled models considered here, as for virtually all present-day wind turbines.

- Boundary layer stability and wind veer due to the Coriolis force. The wind tunnel used in the present research can only general neutrally stable boundary layers. Although atmospheric stability has a profound effect on wakes (Abkara and Porté-Agel, 2015), this problem has already been studied elsewhere, and it is considered to be out of scope for the present investigation. Similarly, Coriolis effects on the inflow and wake behavior are not represented in a wind tunnel, although they are known to have non-negligible effects on capture, loading and also on wake path (van der Laan and Sørensen, 2007). 


\subsection{Neglected quantities}

The following effects could be matched with a different experimental setup and scaling choices, but were neglected in the present work:

- All gravo-aeroelastic effects. Since the blades of the G1 turbine are not aeroelastically scaled (and are very stiff), also the full-scale model was simulated without accounting for flexibility. Aeroelasticity could have some effects on near-wake behavior for very flexible rotors, but would probably have only a negligible role on the characteristics of the far wake. Therefore, aeroelastic effects were excluded from the scope of the present investigation.

- Unsteady airfoil aerodynamics, including linear unsteady corrections (for example, according to Theodorsen's theory (Bisplinghoff and Ashley, 2002)), and dynamic stall. It was verified that the mildly misaligned operating conditions analyzed here would not have triggered dynamic stall, except than in a few instances, similarly to what was found in Shipley (1995). Here again, these effects would hardly have any visible effects on far-wake behavior.

\subsection{Remarks}

Wake stability analysis shows that the vortical structures released by the blade tips and root interact in the near wake (Okulov and Sørensen, 2007).

In the outer shell of the near wake, the mutual interaction of the tip vortices - triggered by turbulent fluctuations and vortex shedding - lead to vortex pairing, leapfrogging, and eventually to the breakdown of the coherent wake structures (Sørensen, 2011). The scaled and full-scale rotors are exposed to the same inflow (including the same turbulent fluctuations), experience the same vortex shedding (due to a matched Strouhal), the tip vortices have the same geometry (due to a matched TSR) and strength (due to a matched circulation), and the speed deficit is also essentially the same (because of the very nearly matched thrust coefficient). Hence, it is reasonable to assume a nearly identical near wake behavior of the external wake shell, given that all main processes are matched between scaled and full-scale models (with the exception of the effects that the unmatched tower may have).

The situation is different in the near wake inner core. Here the root vortices combine with the effects caused by the presence of the nacelle and tower. In particular, the nacelle has a much larger frontal area, creating a different blockage (radial redirection), nacelle wake and vortex shedding. Additionally, in the $20 \%$ inboard portion of the blade, both the circulation and rotational augmentation effects are unmatched. Finally, the mismatch of power induces a mismatch of torque that reduces wake swirl; as it is well known from blade element momentum (BEM) theory, swirl is mostly concentrated in the inner core of the wake, and decays rapidly with radial position (Burton et al., 2011). Hence, the near wake inner core is expected to behave differently in the scaled and full-scale models. However, some of the results reported here, in addition to evidence from other sources (Wu and Porté-Agel, 2011), indicate that the inner core near wake has only a modest effect on far-wake behavior. For example, it is common practice to simulate far-wake behavior with LES codes without even representing the turbine nacelle and tower (Martínez-Tossas et al., 2015). 
As a consequence, thanks to the employed scaling and matching criteria, the far-wake behavior is expected to be extremely similar between the wind tunnel generated wake and the full-scale one. The results section will more precisely support this claim.

\section{Wind turbine models}

\subsection{The TUM G1 scaled wind turbine}

The TUM G1 is a three-bladed clockwise-rotating (looking downstream) wind turbine, with a rotor diameter D of $1.1 \mathrm{~m}$, a hub height $\mathrm{H}$ of $0.825 \mathrm{~m}$, and rated rotor and wind speeds of $850 \mathrm{rpm}$ and $5.75 \mathrm{~ms}^{-1}$, respectively. The $\mathrm{G} 1$ was designed based on the following requirements (Bottasso and Campagnolo, 2020):

- A realistic energy conversion process and wake behavior;

- A sizing of the model obtained as a compromise between Reynolds mismatch, miniaturization constraints, limited wind tunnel blockage and ability to simulate multiple wake interactions within the size of the test chamber;

- Active individual pitch, torque and yaw control in order to test modern control strategies at the turbine and farm levels;

- A comprehensive on-board sensorization.

The turbine has been used for several research projects and numerous wind tunnel test campaigns (Campagnolo et al., 2016, 2020). The main features of the G1 rotor and nacelle are shown in Fig. 1a.

A brushless motor equipped with a precision gearhead and a tachometer is installed in the rear part of the nacelle and provides for the rotor torque, which is in turn measured by a torque sensor located behind the two shaft bearings. An optical encoder, located between the slip ring and the rear shaft bearing, measures the rotor azimuth, while two custom-made load cells measure the bending moments at the foot of the tower and in front of the aft bearing. Thrust is estimated from the tower base fore-aft bending moment, correcting for the drag of the tower and rotor-nacelle assembly.

Each wind turbine model is controlled by its own dedicated real-time modular Bachmann M1 system, implementing supervisory control functions, pitch-torque-yaw control algorithms, and all necessary safety, calibration and data logging functions. Measurements from the sensors and commands to the actuators are transmitted via analogue and digital communication. The Bachmann M1 system is capable of acquiring data with a sample rate of $2.5 \mathrm{kHz}$, which is used for acquiring aerodynamic torque, shaft bending moments and rotor azimuth position. All other measurements are acquired with a sample rate of $250 \mathrm{~Hz}$.

\subsection{Full-scale wind turbine}

A full-scale wind turbine was designed through a backward-engineering approach to match the characteristics of the G1 scaled machine. The DTU 10 MW wind turbine (Bak et al., 2013), shown in Fig. 1b, was used as a starting design for this purpose. This turbine has a rotor diameter of $178 \mathrm{~m}$ and a hub height of $119 \mathrm{~m}$, and the modified version used here is termed G178. 
https://doi.org/10.5194/wes-2020-115

Preprint. Discussion started: 10 November 2020

(C) Author(s) 2020. CC BY 4.0 License.

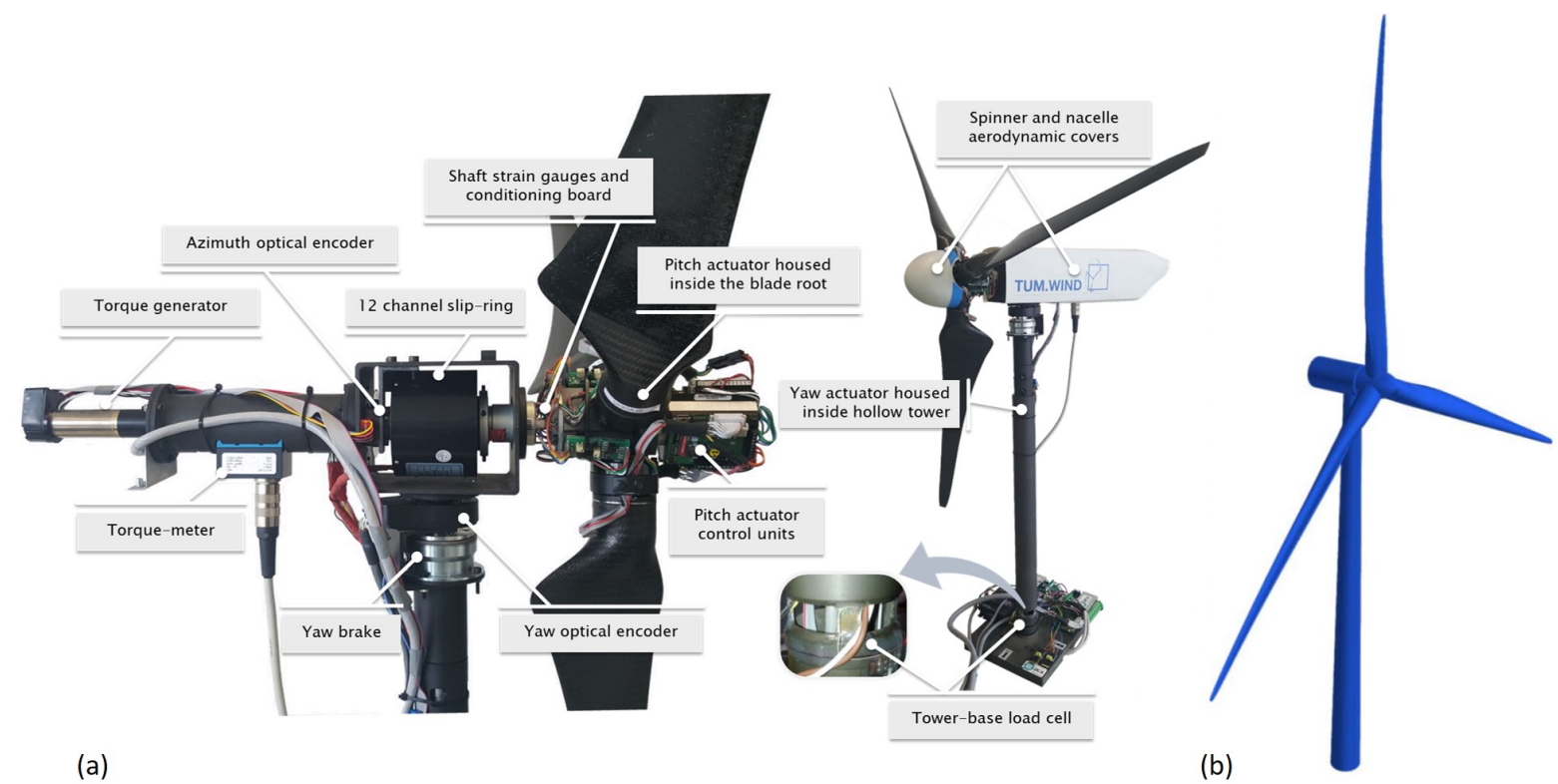

Figure 1. Left: the TUM G1 turbine (Campagnolo et al., 2016). Right: the full-scale DTU 10 MW turbine (from Bak et al. (2013)).

The ratio of the rotor diameter D of the G1 and DTU turbines was used to define the geometric scaling factor $n_{l}$. The hub height $\mathrm{H}$ of the full-scale machine was slightly adjusted to match the ratio $\mathrm{D} / \mathrm{H}$ of the $\mathrm{G} 1$ turbine.

The shape of nacelle and tower were kept the same as the DTU reference, creating a mismatch with the G1 turbine. In fact, the scaled model — due to miniaturization constraints - has a frontal area of the nacelle that is 2.6 times larger than the DTU turbine; similarly, the tower diameter of the G1 turbine is $49 \%$ larger than the DTU machine. This creates a mismatch in the drag of the nacelle and tower, in their local blockage and vortex shedding.

The aerodynamic design of the rotor of the DTU turbine was modified, in order to match the characteristics of the G1 in terms of TSR and circulation distribution (and, as a consequence, also of the thrust). Three versions of the rotor were realized. The standard G178 uses the same airfoils of the DTU turbine over the entire blade span, while chord and twist distributions were modified to satisfy the matching criteria. As the root of the G1 blade is located further away from the rotor axis than in the case of the G178, the circulation is matched only between $20 \%$ and $100 \%$ of blade span. To account for the effects of rotational augmentation, the inboard airfoils were corrected for delayed stall according to the model of Snel (1994).

A second rotor was designed to investigate the effects of the mismatched circulation on wake behavior. To this end, the twist angle close to the root was modified to decrease the lift inboard and match the circulation of the G1 turbine even in this part of the blade; all the other parameters of the model were kept the same of the G178 turbine. This second turbine is termed G178-MC, where MC stands for 'matched circulation'.

A third version of the rotor was obtained by eliminating from the G178 the rotational augmentation model, to investigate its effects. The resulting rotor is termed in the following G178-nRA, where nRA stands for 'no rotational augmentation'. 
Distributions of the twist, chord, lift coefficient and non-dimensional circulation of the G1 and of the full-scale rotors are shown in Fig. 2. Chord distributions are normalized by their respective arithmetic mean values $c_{0}$ over the span. Lift coefficient and circulation are evaluated at rated conditions using the BEM method implemented in the code FAST 8 (Jonkman and Jonkman, 2018). The lift coefficient of the G1 is significantly smaller than the one of the full-scale turbines, which is a result of the low-Reynolds regime of its airfoils. The lower lift is however compensated by a larger chord and different twist distributions, resulting in a matched circulation from $20 \%$ span to the blade tip for the G178 turbine. For the G178-MC model, the circulation is matched over the whole blade span. The difference in lift and circulation between G178 and G178-nRA are due to rotational augmentation.
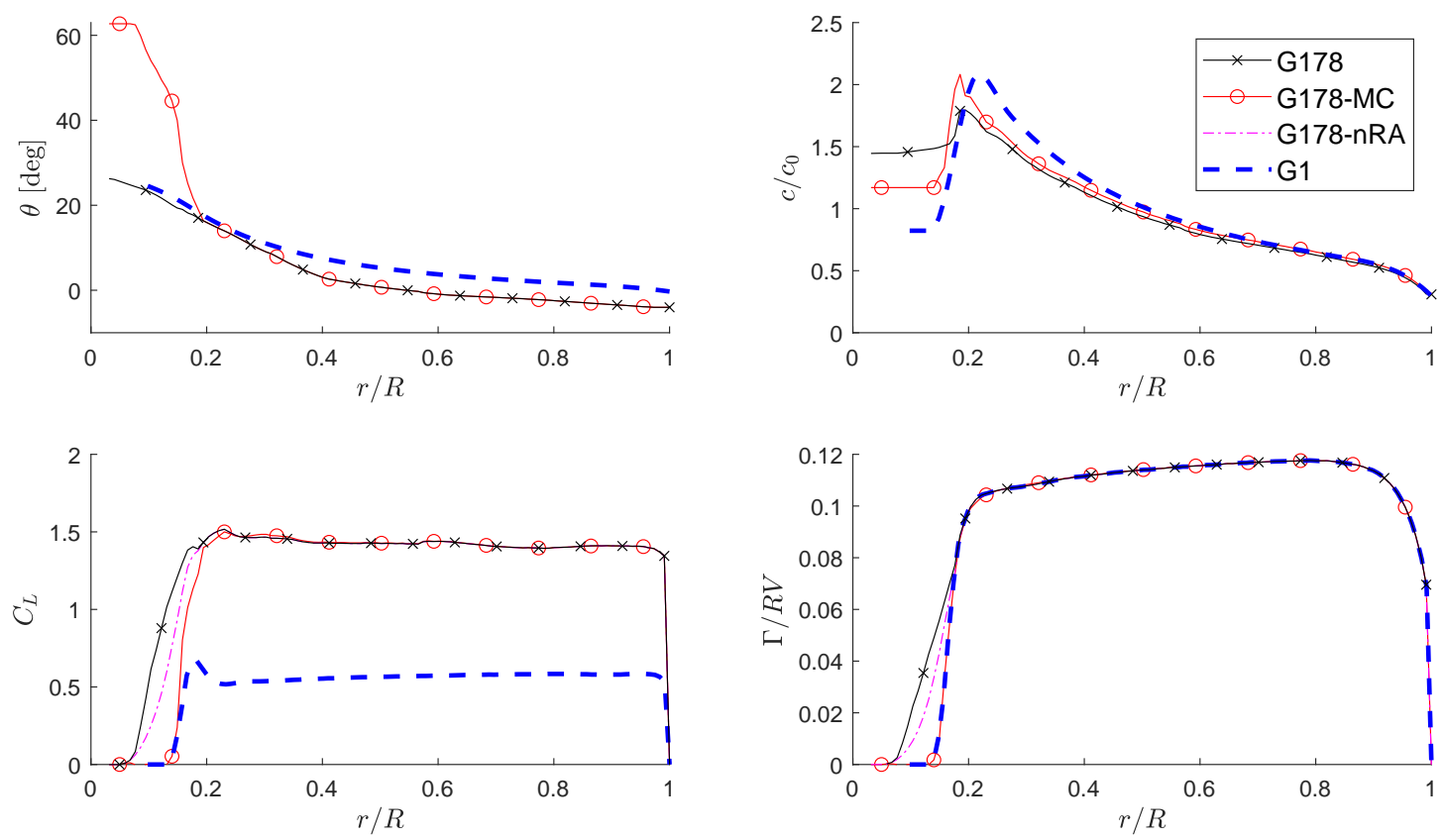

Figure 2. Twist $\theta$, non-dimensional chord $c / c_{0}$, lift coefficient $C_{L}$ and non-dimensional circulation $\Gamma / R V$ distributions for the G1 and for the G178, G178-MC and G178-nRA full-scale turbines.

\section{Simulation model}

\subsection{LES-ALM CFD code}

Numerical results were obtained with a TUM-modified version of SOWFA (Fleming et al., 2014), more completely described in Wang et al. (2018, 2019). The code has been used extensively to numerically replicate wind tunnel tests conducted with G1 
turbines, achieving an excellent correlation with the experimental measurements in a wide range of conditions, including full and partial wake overlaps, wake deflection, static and dynamic induction control, and individual pitch control (for example, see Wang et al. (2019, 2020b, c)).

The finite volume LES solver is based on the standard Boussinesq PISO (Pressure Implicit with Splitting of Operator) incompressible formulation, and is implemented in OpenFOAM (Jasak, 2009). Spatial differencing is based on the Gamma method (Jasak et al., 1999), where a higher level of upwinding is used in the near wake region to enhance stability. Time marching is based on the backward Euler scheme. The pressure equation is solved by the conjugate gradient method, preconditioned by a geometric-algebraic multi-grid, while a bi-conjugate gradient is used for the resolved velocity field, dissipation rate and turbulent kinetic energy, using the diagonal incomplete-LU factorization as preconditioner. The turbulence model is based on the Constant Smagorinsky method (Smagorinsky, 1963).

An actuator-line method (ALM) (Troldborg et al., 2007) is used to represent the effects of the blades, according to the velocity sampling approach of Churchfield et al. (2017). The implementation of the actuator lines is obtained by coupling the CFD solver with the aeroservoelastic simulator FAST 8 (Jonkman and Jonkman, 2018). For improved accuracy, the airfoil polars of the G1 are tuned based on experimental operational data (Bottasso et al., 2014b; Wang et al., 2020a).

Finally, an immersed boundary (IB) formulation method (Mittal and Iaccarino, 2005; Jasak and Rigler, 2014) is employed to model the effects of the turbine nacelle and tower.

\subsection{Turbulent inflow}

Experiments with the G1 turbine took place in the large boundary layer test section of the wind tunnel at the Politecnico di Milano, where a turbulent flow is generated passively by the use of trapezoidal spires. Without the spires, the flow at the inlet has a turbulence intensity (TI) of about $1-2 \%$ and a small horizontal variability caused by the presence of 14 fans and internal transects upstream of the chamber. The non-uniform blockage caused by the spires decelerates the flow close to the wind tunnel floor, generating an initial vertical shear; furthermore, large vortical structures develop around the edges of the spires, which then break down as the flow evolves moving downstream.

Two setups are considered, with two different TI levels. To mimic a typical medium-turbulence offshore condition, 14 type-B spires were placed side by side $1 \mathrm{~m}$ from each other, $1 \mathrm{~m}$ downstream of the test chamber inlet. A type-B spire consists of an equilateral trapezoid and a supporting board. The height of the trapezoid is $2.0 \mathrm{~m}$, while the widths of the bottom and top edges are $0.26 \mathrm{~m}$ and $0.1 \mathrm{~m}$, respectively. The developed turbulent flow where the turbine is located $(19.1 \mathrm{~m}$ downstream of the inlet) has a vertical shear with a power coefficient equal to 0.12 , a small horizontal shear and hub-height speed and TI of $5.75 \mathrm{~ms}^{-1}$ and 5\%, respectively. A second higher-turbulence inflow was generated using 9 spires of $2.5 \mathrm{~m}$ of height, a base of $0.8 \mathrm{~m}$, placed at a distance of $1.55 \mathrm{~m}$ from each other. In addition, 24 rows of $0.23 \times 0.23 \times 0.1 \mathrm{~m}$ bricks were placed on the ground, with 12 bricks in odd rows and 13 bricks in even ones, resulting in a staggered brick distribution. This second configuration resulted in a vertical shear with a power coefficient equal to 0.19 , a small horizontal shear, and hub-height speed and TI of $5.75 \mathrm{~ms}^{-1}$ and $14 \%$, respectively. 
The same process of passive turbulence generation was simulated by using the LES code. The mesh was generated with ANSYS-ICEM, obtaining a structured body-conforming grid around the spires (Wang et al., 2019), while the bricks placed on the floor for the higher turbulence case were modelled by the IB method. Figure 3 shows the mean streamwise velocity distribution at the chamber cross-section 3.57 D in front of the rotor. The plots on the left report the results of an experimental mapping of the flow performed with triple hot wire probes, while the ones on the right report the numerical results for the medium (top row) and high (bottom row) turbulence cases; notice that measurements are available only $0.2 \mathrm{~m}$ above the floor. A good match between experimental measurements and simulation results can be observed over the whole cross-section of the test chamber, including not only the vertical shear but also the slight horizontal non-uniformities.
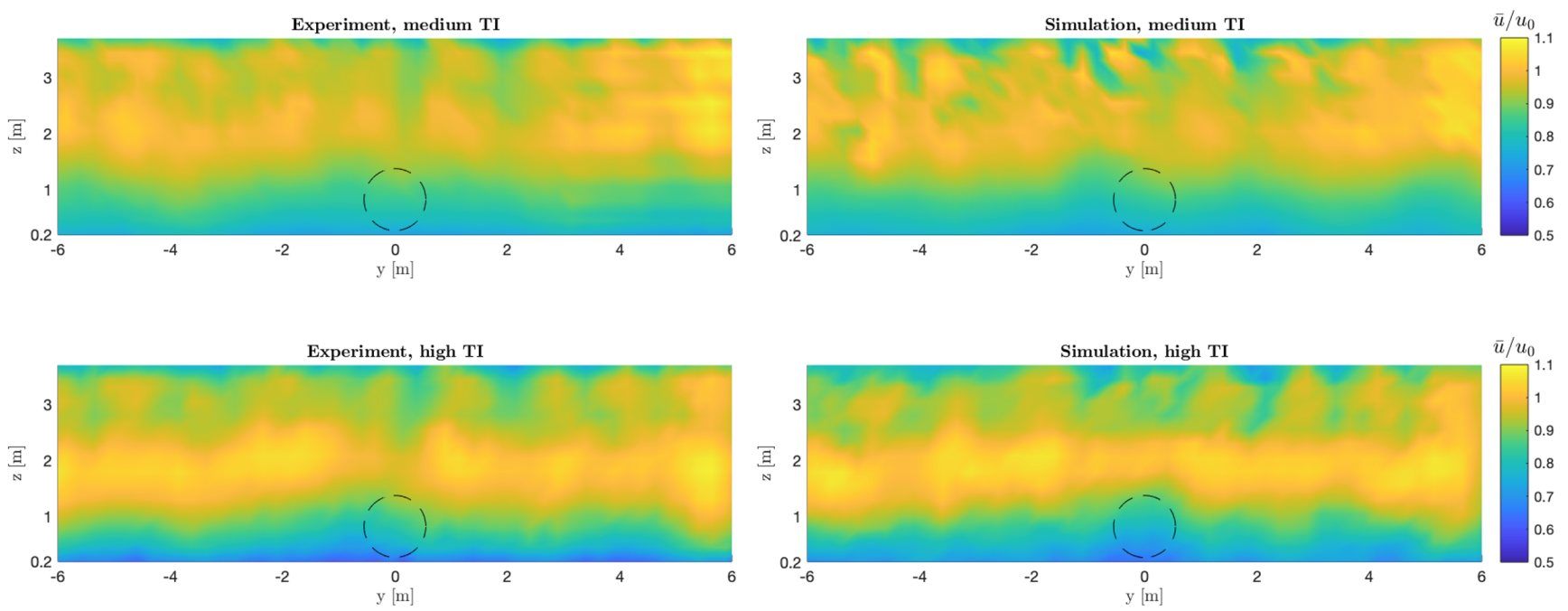

Figure 3. Streamwise velocity distribution on a cross section of the test chamber 3.57 D in front of the rotor. Left: experimental measurements; right: numerical simulation; top: medium TI case; bottom: high TI case.

For the same plane, Fig. 4 shows the mean (i.e., time-averaged) speed and TI profiles along a vertical line directly in front of the rotor center. Here again, a good match between experimental measurements and simulation can be observed, except in the immediate proximity of the floor.

The results of the passive turbulence-generating precursor simulations were sampled on the plane $3.57 \mathrm{D}$ upstream of the turbine, and used as inlet for the simulations of the turbine and its wake, including the side walls and the ceiling of the tunnel. The chamber cross section has a width of $13.84 \mathrm{~m}$ and a height of $3.84 \mathrm{~m}$, resulting in some vertical blockage, whose effects were quantified by running various simulations for increasing values of the chamber height, as reported later. The wind tunnel grid uses three zones of increasing density, the smallest cells having a size of $0.015 \mathrm{~m}$ (i.e., $1.4 \cdot 10^{-2} \mathrm{D}$ ). The ALM discretization used 108 points over the blade span, i.e. a spacing equal to $4.7 \cdot 10^{-3} \mathrm{D}$.

For the full-scale machine, each inflow was scaled in space and time, as previously explained, resulting in flows with the same identical characteristics at the two scales. Similarly, the same LES and ALM grids were geometrically upscaled and used 

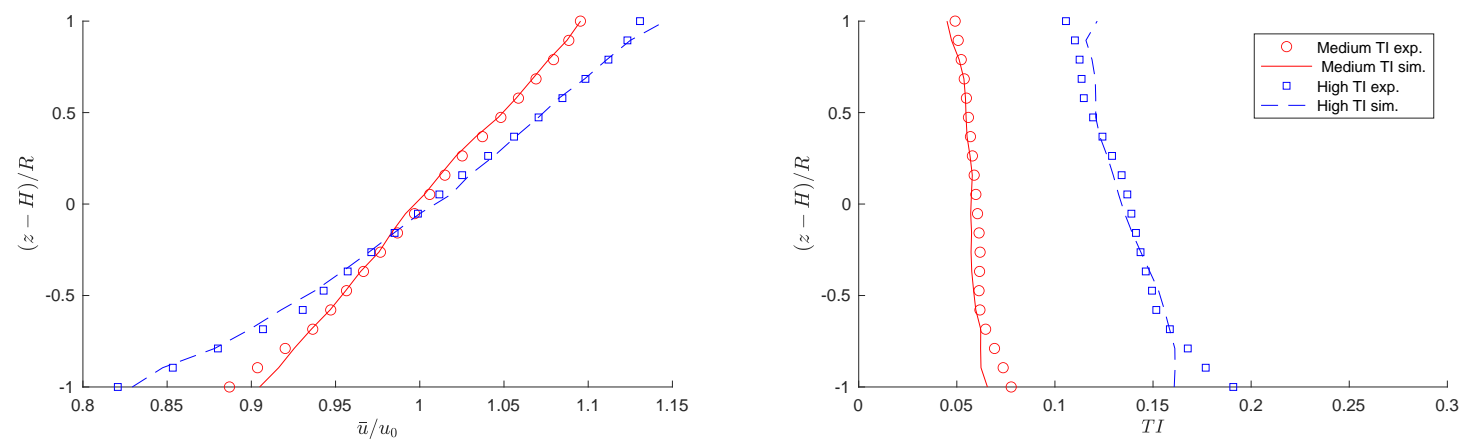

Figure 4. Mean velocity (left) and turbulence intensity (right) distributions along a vertical line 3.57 D in front of the rotor.

for the full-scale simulations; this means that also the full-scale simulations have the same slight anisotropic blockage effects of the wind tunnel case.

\section{Results}

\subsection{Code to experiment verification}

First, experimental measurements obtained with triple hot wire probes are compared with the corresponding numerical simulations. Two operating conditions in the partial load regime (region II) are considered: one aligned with the flow and one with a misalignment angle $\gamma$ of $20 \mathrm{deg}$. Table 1 reports the experimental and simulated power and thrust coefficients in the two cases, in medium TI conditions. Figure 5 reports a comparison of horizontal scans of the wake (Wang et al., 2019) for the aligned case at various downstream distances for both the medium and high TI cases.

Table 1. Experimental and simulated power and thrust coefficients for the G1 turbine, in the medium TI Case.

\begin{tabular}{|c|cc|cc|}
\hline \hline \multirow{2}{*}{ Coefficient } & \multicolumn{2}{|c|}{$C_{P}$} & \multicolumn{2}{c|}{$C_{T}$} \\
\hline Case & Experiment & Simulation & Experiment & Simulation \\
\hline$\gamma=0 \mathrm{deg}$ & 0.416 & 0.420 & 0.881 & 0.851 \\
\hline$\gamma=20 \mathrm{deg}$ & 0.364 & 0.358 & 0.810 & 0.742 \\
\hline \hline
\end{tabular}

The figure shows hub-height horizontal time-average streamwise velocity (top panel) and turbulence intensity (bottom panel) profiles. While the match of the wake profile is excellent for all locations, the numerical results slightly overestimates turbulence intensity in the center of the near wake region. Overall, simulation and experimental results are in very good agreement. 

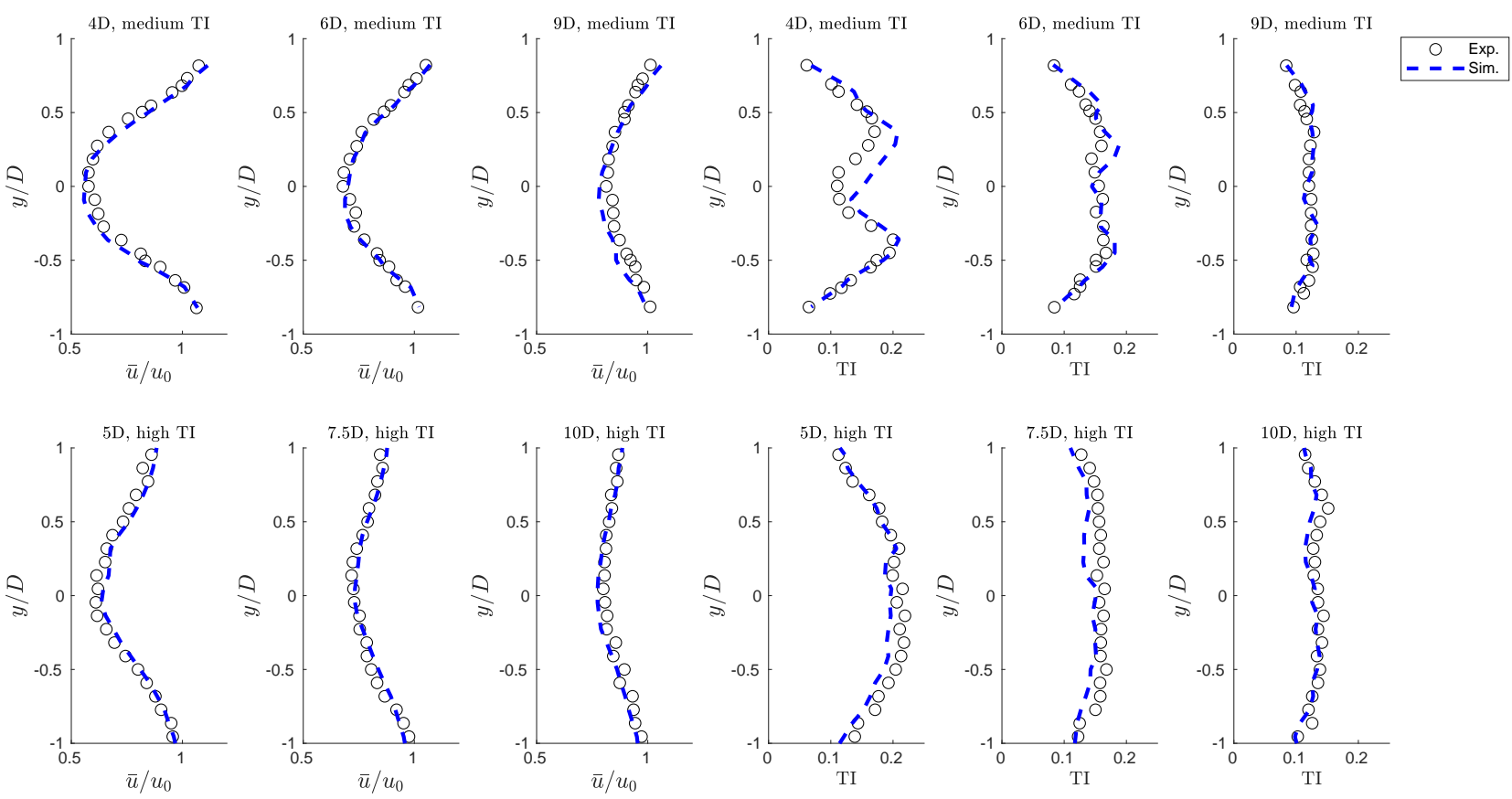

Figure 5. Horizontal hub-height profiles of normalized time-average streamwise velocity and turbulence intensity, for the medium (top) and high (bottom) inflow TI cases. Black o symbols: experimental results; blue dashed line: G1 simulations.

\subsection{Scaled to full-scale comparisons}

Next, having established a good correspondence between the numerical results and experimental measurements, simulations were conducted with the full-scale turbines to understand the effects of mismatched quantities.

Table 2 shows the turbine power and thrust coefficients for the different cases, considering the G1 and three G178 turbine models. As expected, the power coefficient of the G1 turbine is lower than the one of all full-scale G178s, because of the lower efficiency caused by the different Reynolds regime. On the other hand, there is a good match of the thrust coefficient, especially for G178; the nRA and MC versions produce a slightly lower lift in the inboard section of the blade, and hence have a marginally lower $C_{T}$.

Figure 6 gives a qualitative overview of the wakes of the G1 and G178 turbines for the aligned and misaligned cases. The wake deficits are similar, except for the central region of the near wake, as expected. Even this qualitative view shows a significant effect of the much larger nacelle of the G1. This difference however disappears moving downstream, and the far wakes of two turbines appear to be almost identical. 
Table 2. Power and thrust coefficients for the different turbine models in the two considered operating conditions.

\begin{tabular}{|c|cccc|cccc|}
\hline \hline Coefficient & \multicolumn{3}{|c|}{$C_{P}$} & \multicolumn{4}{c|}{$C_{T}$} \\
\hline Turbine model & G1 & G178 & G178-nRA & G178-MC & G1 & G178 & G178-nRA & G178-MC \\
\hline$\gamma=0$ deg & 0.420 & 0.475 & 0.472 & 0.470 & 0.851 & 0.831 & 0.827 & 0.822 \\
\hline$\gamma=20 \mathrm{deg}$ & 0.358 & 0.421 & 0.418 & 0.417 & 0.742 & 0.731 & 0.727 & 0.723 \\
\hline \hline
\end{tabular}
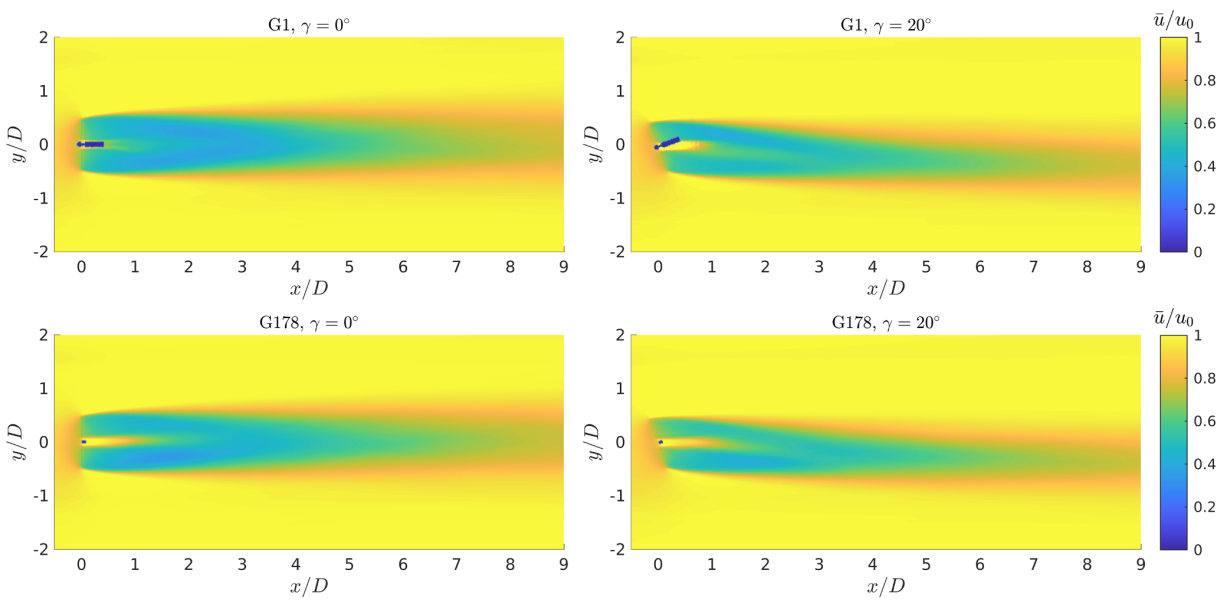

Figure 6. Wakes of the scaled G1 and full-scale G178 turbines. Left: aligned case; right: yaw misaligned case.

A more precise characterization of the differences between the scaled G1 model and the realistic full-scale G178 turbine is given by Fig. 7 (medium TI) and 8 (high TI), considering the misaligned case. For both figures, the first row shows the mean speed in the longitudinal direction, while the second and third rows show the Reynolds shear stress components $\overline{u^{\prime} u^{\prime}} / u_{0}^{2}$ and $\overline{u^{\prime} v^{\prime}} / u_{0}^{2}$, respectively, where the prime here indicates a fluctuation with respect to the mean.

Results indicate an excellent match between the scaled and full-scale wakes, for both TI levels. Some differences only appear in the peaks of $\overline{u^{\prime} u^{\prime}} / u_{0}^{2}$ immediately downstream of the rotor. However, the velocity profiles are remarkably similar already at $3 \mathrm{D}$, notwithstanding the differences around the hub and blade inboard sections between the two machines. Similar conclusions are obtained for the aligned case.

\subsection{Effects of unmatched inboard circulation and rotational augmentation}

The effects of unmatched inboard circulation and rotational augmentation are quantified by computing the differences in $\bar{u} / u_{0}$, $\overline{u^{\prime} u^{\prime}} / u_{0}^{2}$ or $\overline{u^{\prime} v^{\prime}} / u_{0}^{2}$ at different downstream locations. Results are shown in Fig. 9, where differences are computed subtracting the G178 solution from the G178-MC or G178-nRA ones. As indicated by the figure, these effects are extremely small, and possibly discernible from numerical noise only in the immediate proximity of the rotor. 
https://doi.org/10.5194/wes-2020-115

Preprint. Discussion started: 10 November 2020

(c) Author(s) 2020. CC BY 4.0 License.

eawế
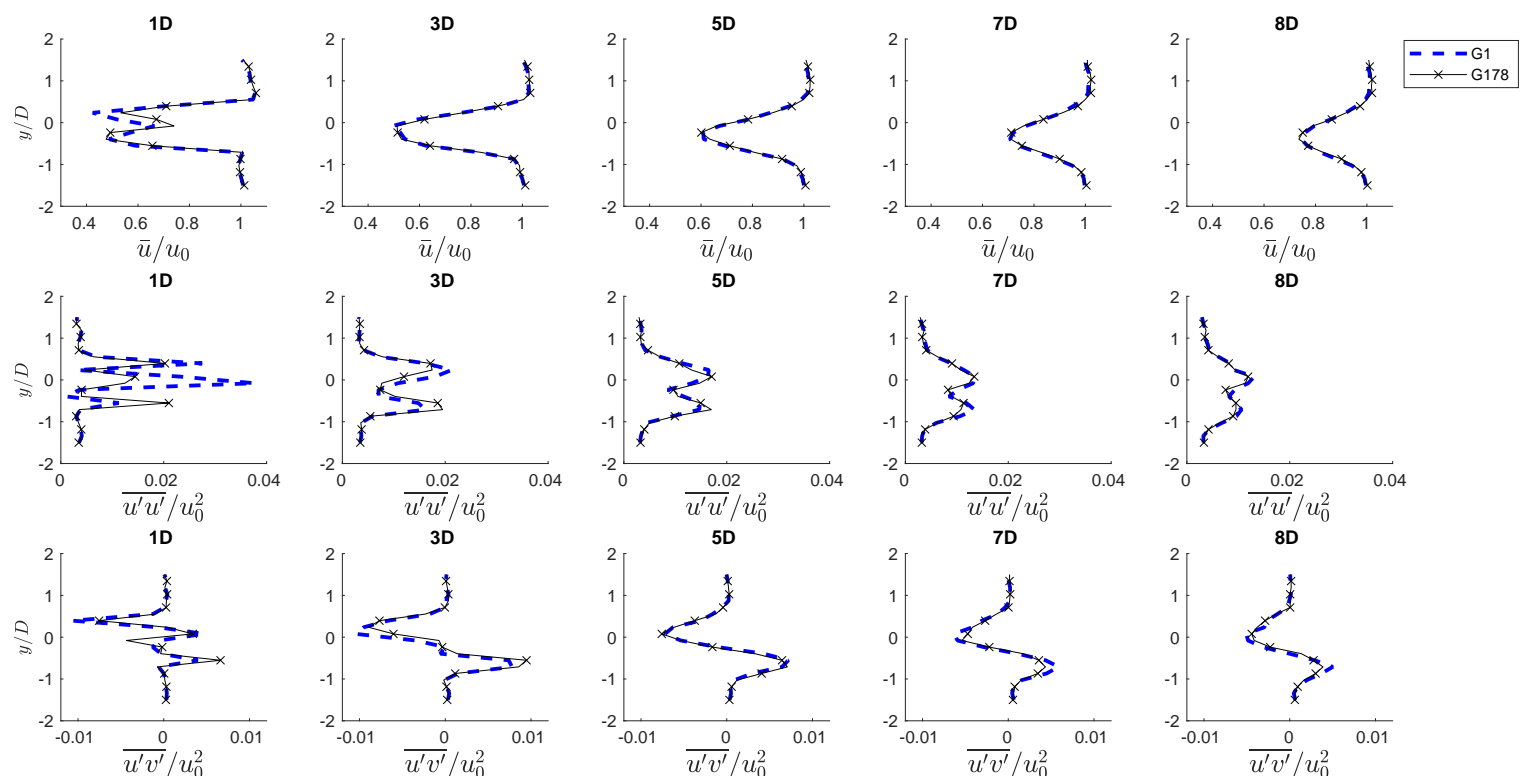

Figure 7. Hub-height profiles of normalized time-average streamwise velocity (top) and shear stresses (center and bottom), in the misaligned and medium TI condition.
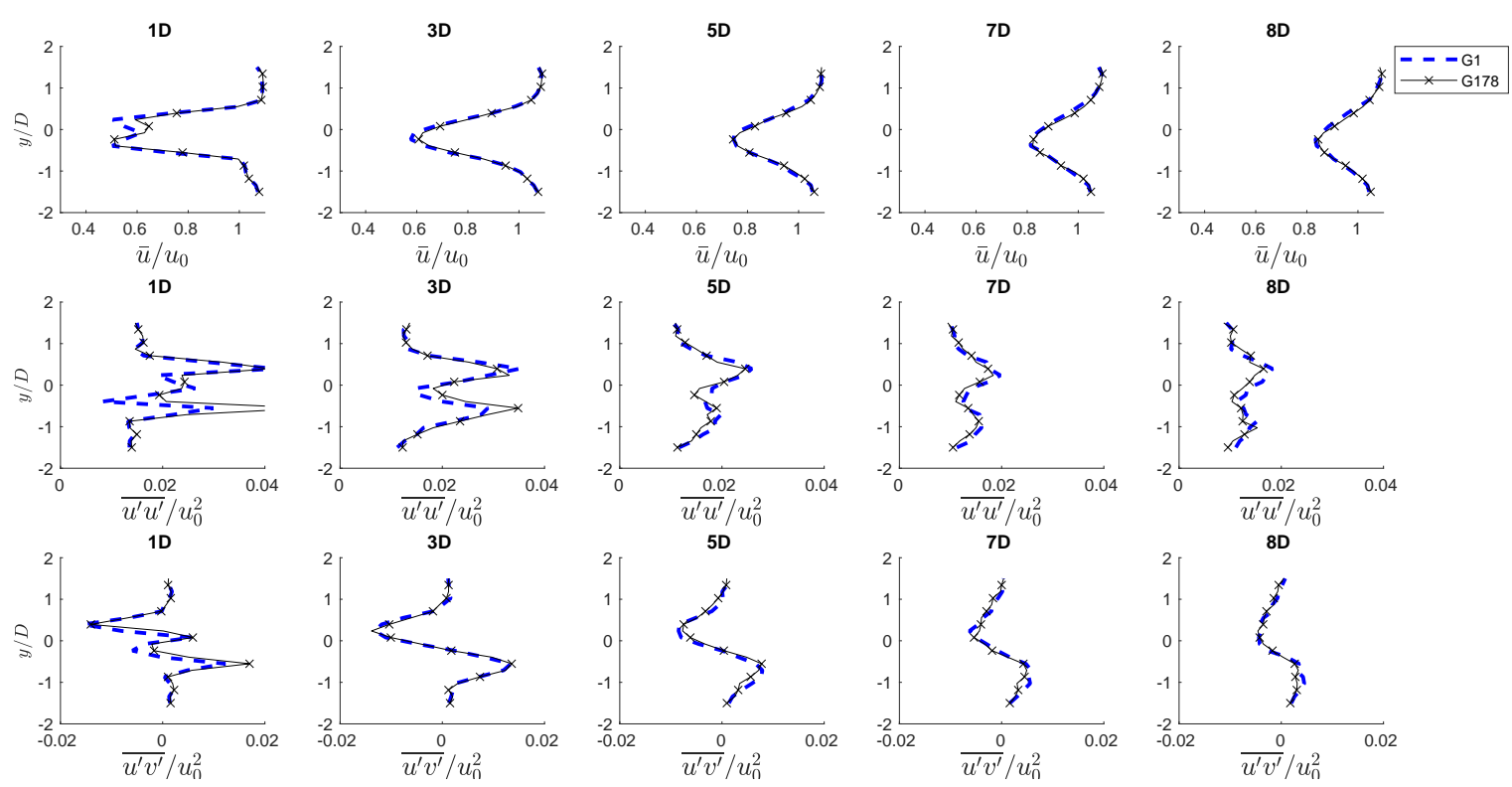

Figure 8. Hub-height profiles of normalized time-average streamwise velocity (top) and shear stresses (center and bottom), in the misaligned and high TI condition. 

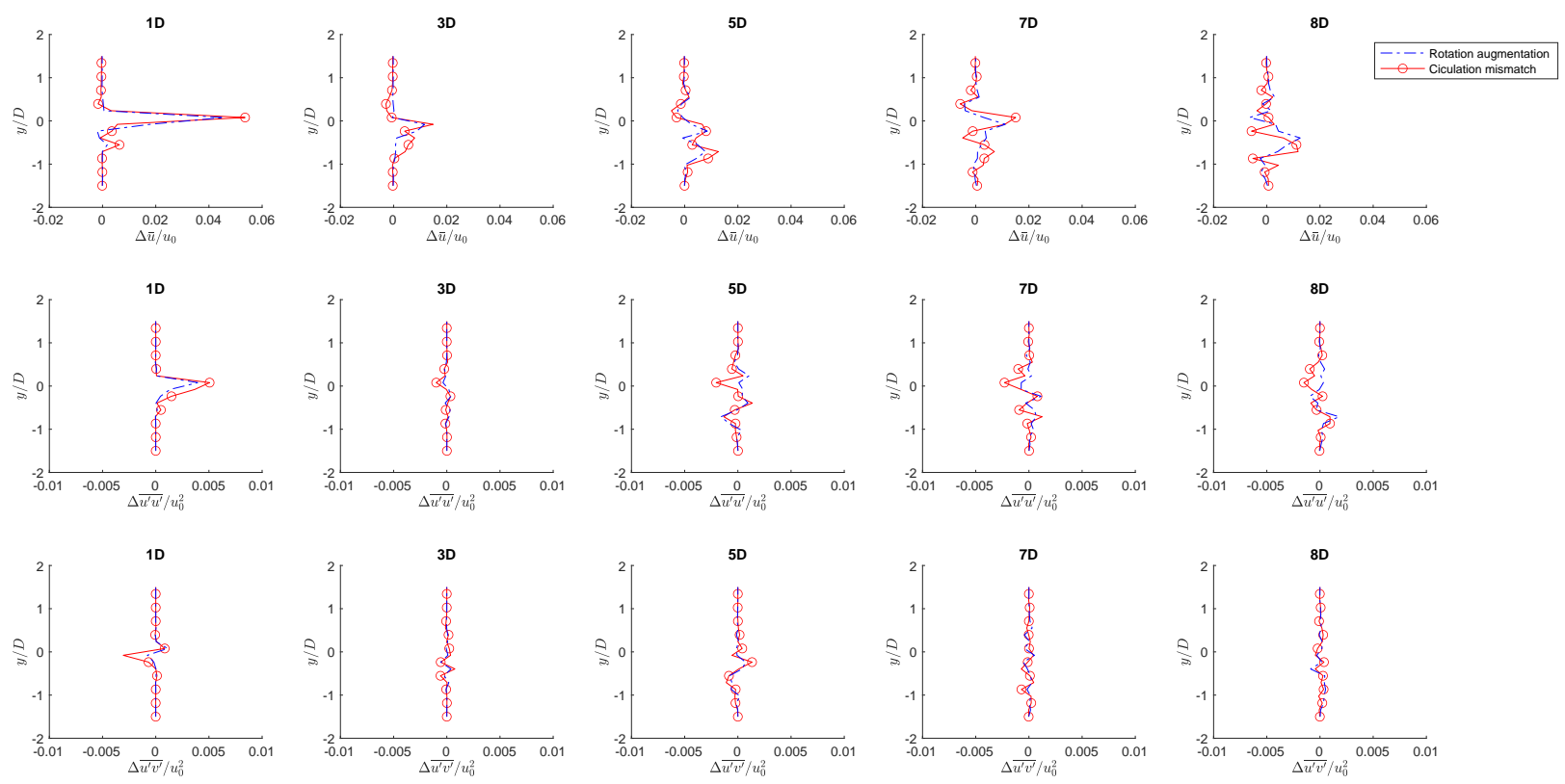

Figure 9. Difference in the profiles of the normalized time-average streamwise velocity (top) and shear stresses (center and bottom) along hub-height horizontal lines, caused by rotation augmentation (dash-dotted blue line) and by a mismatched circulation close to the root (red solid line and o symbols). Results are for the yaw misaligned and medium TI condition.

\subsection{Effect of nacelle size and unmatched $C_{P}$ on swirl}

For the wind-aligned operating condition, Fig. 10 shows the delta wake velocity field obtained by subtracting the G178-MC from the G1 solution, looking upstream. The panel on the left represents the near wake $1 \mathrm{D}$ immediately behind the rotor disk plane, while the panel on the right reports the far wake at $8 \mathrm{D}$. The color field represents the difference in the non-dimensional streamwise velocity component $\Delta\left(\bar{u} / u_{0}\right)$, whereas the arrows represent differences in the in-plane velocity vectors.

In this case, since the circulation is matched, there are only two factors that could result in non-zero difference fields: the larger frontal area of the nacelle (and, similarly, of the tower) of the G1, and its smaller power coefficient caused by the Reynolds mismatch. The impacts of these two factors are clearly visible in the near wake, respectively looking at the streamwise and in-plane velocities.

In fact, the negative streamwise velocity bubble at the center of the rotor is a result of the larger blockage of the G1 nacelle. The effect of the tower differs from that of the nacelle. While the nacelle is almost a pure blockage in the center of the rotor where wake recovery is the weakest, the presence of the tower wake can increase the local turbine wake recovery by increasing turbulence intensity. As the wake rotates counter-clockwise when looking upstream as in Fig. 10, the flow influenced by tower is also convected towards the negative $y$ direction. 

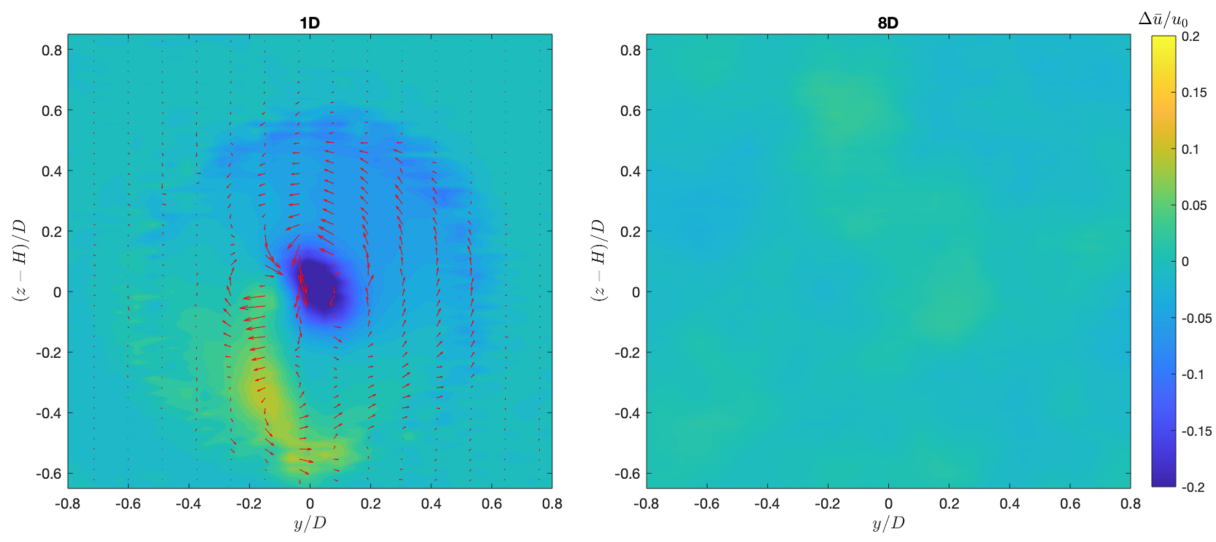

Figure 10. Difference in the wake velocity fields between the G1 and the G178-MC turbines, looking upstream. Color field: non-dimensional streamwise velocity difference $\Delta\left(\bar{u} / u_{0}\right)$; arrows: difference in the in-plane velocity vectors. Left: near wake $1 \mathrm{D}$ immediately behind the rotor disk plane; right: far wake at $8 \mathrm{D}$.

When looking upstream, the rotor spins counterclockwise, whereas the wake rotates clockwise by the principle of action and reaction. Compared to the wake of the G178-MC turbine, the wake of the G1 rotates at a slower pace, as indicated by the counterclockwise rotation of the difference field shown in the picture. The slower rotation of the G1 wake is a direct consequence of its smaller power coefficient that, for the same TSR, implies also a reduced torque coefficient. As expected, the mismatch in the swirl rotation is only concentrated close to the hub, and decays quickly with radial position.

As the flow propagates downstream and the wake progressively recovers, differences between the velocity fields decay and the effects of the mismatches can hardly be seen at $8 \mathrm{D}$. The only difference that can still be identified is the effect of the larger tower. This results in some blockage close to the ground that has not yet fully recovered at this distance, resulting in about a $6 \%$ difference in the longitudinal velocity component immediately above the floor and, hence, in a slightly enhanced shear below hub height. Elsewhere, differences between the two fields never exceed 3\%.

\subsection{Effect of wind tunnel blockage}

Considering the G1 turbine, the wind tunnel test chamber has a height $h_{w t}=3.49 \mathrm{D}$ and a width $w_{w t}=12.49 \mathrm{D}$, resulting in a cross sectional area $A_{w t}=43.59 \mathrm{D}$. Although the resulting area ratio $A_{w t} / A=55.5$ is relatively large, the small vertical ratio $h_{w t} / D$ can cause some anisotropic blockage. To quantify this effect, numerical simulations were conducted in domains of increasing height from 1.75 D to $10.47 \mathrm{D}$, as shown in the left panel of Fig. 11. The actual wind tunnel height is indicated by a red square mark in the figure.

The right panel of Fig. 11 shows the non-dimensional power increase $\Delta P / P_{\infty}$ vs. the area ratio $A_{w t} / A$, where $P_{\infty}$ is the power for the largest domain — assumed to be blockage-free. Results indicate a power increase caused by blockage of about $1.5 \%$. 

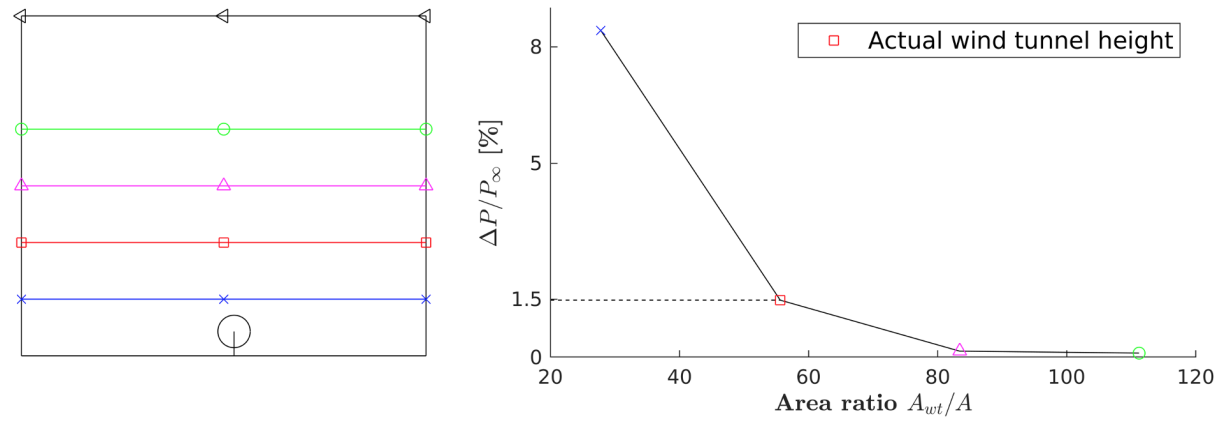

Figure 11. Wind tunnel blockage effect. Left: cross sectional areas; right: percent power increase with respect to the unrestricted flow.

\subsection{Wind farm control metrics}

The previous analysis has shown that the wake of the G1 turbine has a very close resemblance to the one of the full-scale G178, although some differences are present in the near wake region. However, it is difficult to appreciate the actual relevance of these differences, and a more practical quantification of the accuracy of the match would be desirable. The G1 turbine is mostly used for studying wake interactions within clusters of turbines, and for testing mitigating control strategies. This suggests the use of wind-farm-control-inspired metrics for judging the differences between the scaled and full-scale machines.

The first metric considered here is the available power ratio $P_{a}(x / D) / P_{0}=\bar{V}^{3}(x / D) / V_{\infty}^{3}$ downstream of the turbine, where $P_{0}$ is the power output of the turbine, $V_{\infty}$ is the ambient wind speed at hub height, and $\bar{V}(x / D)$ is the rotor-effective wind speed at the downstream location $x / D$. The available power ratio depends on the shape of the wake, its recovery and trajectory, and it was computed from the longitudinal flow velocity component in the wake on the area of the rotor disk at various downstream positions directly behind the wind turbine, as shown in Fig. 12.

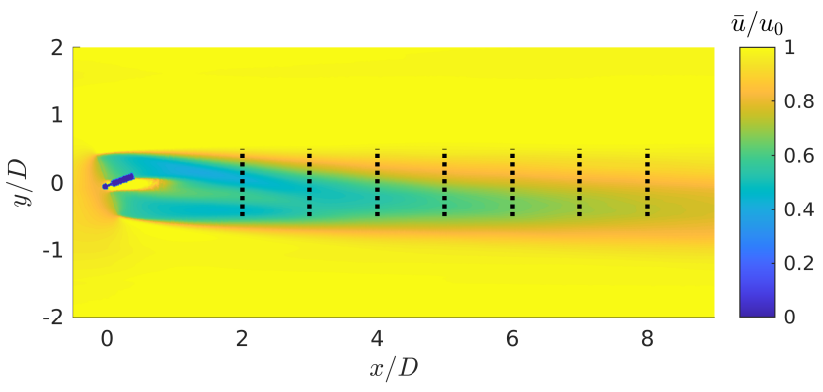

Figure 12. Wake of the G1 turbine for the yaw misaligned case. The black dashed lines indicate the locations of virtual downstream turbines.

For the 20 deg misaligned case, the available power ratio results are shown in the left panel of Fig. 13. As shown in the figure, the available power changes moving downstream because the wake expands, recovers and — since the turbine is misaligned 
with respect to the wind vector - shifts progressively more to the side of the impinged (virtual) rotors. The difference of the available power behind the G1 and G178 turbines is small, and decreases quickly moving downstream. The figure also shows the effects of blockage, by reporting the results for the actual wind tunnel size using a solid line, and the ones for the unrestricted case using a dashed line; here again, this effect is very modest.
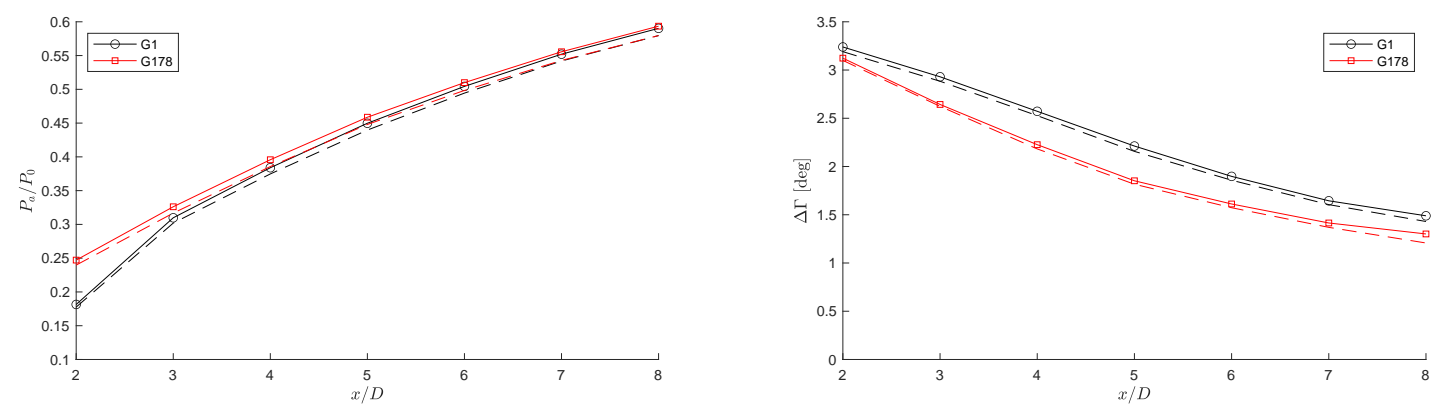

Figure 13. Left: available power ratio in the wake $P_{a} / P_{0}$ as a function of downstream position $x / D$. Right: change of wind direction $\Delta \Gamma$ caused by the curled wake as a function of downstream position $x / D$. Both results are for the 20 deg misaligned and medium TI case. Black o symbols: G1; red $\square$ symbols: G178. Solid lines: actual wind tunnel size; dashed lines: unrestricted case (no blockage).

The second metric considered here is the ambient flow rotation in the immediate proximity of a deflected wake. By misaligning a wind turbine rotor with respect to the incoming flow direction, the rotor thrust force is tilted, thereby generating a cross-flow force that laterally deflects the wake. As shown with the help of numerical simulations by Fleming et al. (2018), this cross-flow force induces two counter rotating vortices that, combining with the wake swirl induced by the rotor torque, lead to a curled wake shape. As observed experimentally by Wang et al. (2018), the effects of these vortices result in additional lateral flow speed components, which are not limited to the wake itself but extend also outside of it. By this phenomenon, the flow direction within and around a deflected wake is tilted with respect to the upstream undisturbed direction. Therefore, when a turbine is operating within or close to a deflected wake, its own wake undergoes a change of trajectory — termed secondary steering — induced by the locally modified wind direction.

The change in ambient wind direction $\Delta \Gamma$ caused by the curled wake is reported in the right panel of Fig. 13 as a function of the downstream distance $x / D$; even in this case, the effects of blockage can be appreciated by comparing the solid and dashed lines. The angle $\Delta \Gamma$ was computed from the wake velocity components, averaging over the rotor disk areas already used for the analysis of the available power. Here again the difference in the change of ambient wind direction behind the G1 and G178 turbines is quite small. A non-perfect match is probably due to the slightly different strength of the central vortex generated in response to the rotor torque. On the other hand, the two counter-rotating vortices caused by the tilted thrust are well matched — given the good correspondence of this force component between the two models. 
https://doi.org/10.5194/wes-2020-115

Preprint. Discussion started: 10 November 2020

(c) Author(s) 2020. CC BY 4.0 License.

\section{(c) (1)}

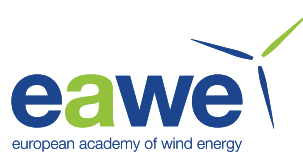

\subsection{Effect of integral length scale}

The ILS of the wind tunnel flow was obtained by first computing the time-autocorrelation of the wind speed at one position in front of the turbine, and then multiplying the result by the mean wind speed. The length scales obtained from measurements in the wind tunnel and the simulated flow resulted in nearly identical values, as already shown by Wang et al. (2019). A second estimate of the ILS was based on the space-autocorrelation between simultaneous values of the simulated wind speed at two points in front of the turbine. For the size of the G1 turbine, this second estimate of the ILS resulted in a full-scale value of approximatively $142 \mathrm{~m}$. On the other hand, the IEC 61400-1 international standards prescribe space-autocorrelation-based lengths of $170 \mathrm{~m}$ in Ed. 2 (IEC 61400-1, 1999) and of $340 \mathrm{~m}$ in Ed. 3 (IEC 61400-1, 2005). Although the ILS presents a significant natural variability at each location and across different sites (Kelly, 2018), the value achieved in the wind tunnel with the G1 is undoubtedly in the low range of naturally occurring scales.

To understand the effects of the partially mismatched ILS on wake behavior, two turbulent inflows were generated, differing only in this parameter. Unfortunately, however, the natural development of two inflows with different ILS values but exactly the same TI and vertical shear is clearly an extremely difficult task. To avoid this complication, the code TurbSim was used, selecting the Kaimal model and prescribing directly the turbulence scale parameter (see Eq. (23) in Jonkman (2009)). The resulting turbulent wind time histories were specified as Dirichlet inflow conditions for the subsequent LES-ALM simulations.

The two resulting developed CFD flows are characterized by an ILS of $176 \mathrm{~m}$ and $335 \mathrm{~m}$, and have a vertical shear exponent 0.18 and hub-height speeds and TI of $11.3 \mathrm{~ms}^{-1}$ and $6.0 \%$, respectively. These two different flows were used for conducting dynamic simulations with the G178 turbine in a 20 deg yaw misaligned condition.

The ILS indicates the dimension of the largest coherent eddies in the flow. Hence, the main effect of a larger ILS is that of inducing a more pronounced meandering of the wake. To quantify this effect, the instantaneous wake center was computed according to the deficit-weighted center of mass method (España et al., 2011). The standard deviation of the horizontal wake position $5 \mathrm{D}$ downstream of the rotor was found to be equal to $0.089 \mathrm{D}$ for the low ILS (176 m) case, and equal to $0.12 \mathrm{D}$ for the high ILS (335 m) one, according to expectations.

The effects of a different ILS are much smaller, although still appreciable, when considering mean quantities. Figure 14 reports the profiles of speed and shear stresses at different downstream distances. The mean velocity profile is only very slightly affected, with a maximum change of about only $2 \%$. A clearer effect is noticeable in the shear stresses at the periphery of the wake.

\section{Conclusions}

This paper has analyzed the realism of wind-tunnel-generated wakes with respect to the full-scale case. In the absence of comparable scaled and full-scale experimental measurements, a hybrid experimental-simulation approach was used here for this purpose. A LES-ALM code was first verified with respect to detailed measurements performed in a large boundary layer wind tunnel with the TUM G1 scaled wind turbine. Next, the same code — with the same exact algorithmic settings— was 

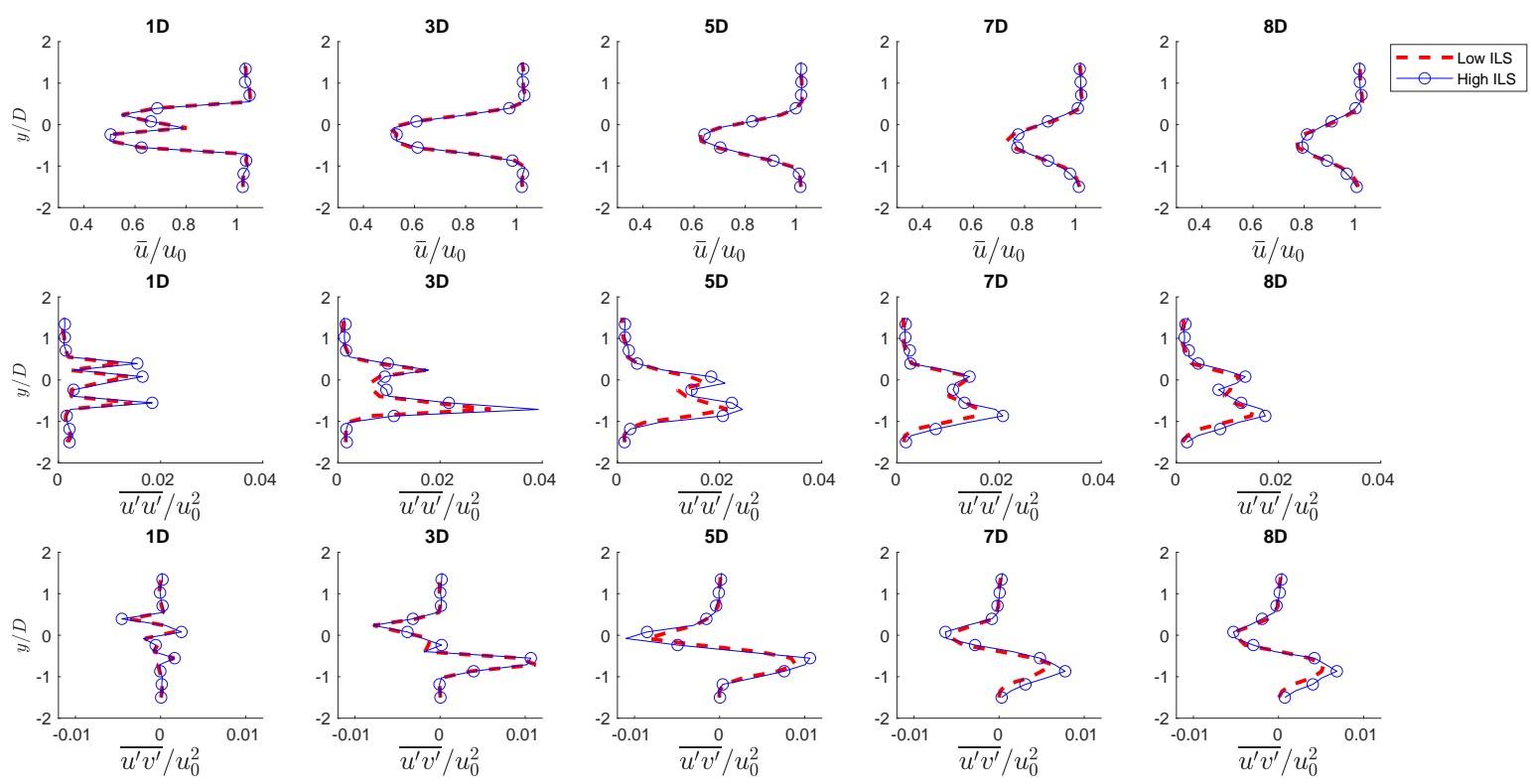

Figure 14. Hub-height profiles of normalized time-average streamwise velocity (top) and shear stresses (center and bottom), for the low and high ILS cases in yaw misaligned conditions.

used to simulate different full-scale versions of the scaled turbine. These different full-scale models were designed to highlight the effects of mismatched quantities between the two scales.

Clearly, this approach has some limits and therefore falls short of providing a comprehensive answer to the realism question.

In fact, the comparison is clearly blind to any physical process that is not modelled or that is not accurately resolved by the numerical simulations. Additionally, it is assumed that a numerical model that provides good quality results with respect to reality at the small scale is also capable of delivering accurate answers at the full scale.

Keeping in mind these limits, the following conclusions can be drawn from the present study:

- Overall, the far (above approximatively 4 D) wake of the G1 scaled wind turbine is extremely similar to the wake of a corresponding full-scale machine considering all classical mean metrics, i.e. wake deficit, turbulence intensity, shear stresses, wake shape and path, both in aligned and misaligned conditions.

- Small differences of fractions of a degree are present in the local wind direction changes caused by the curled wake, because of a different swirl generated by the lower aerodynamic torque of the scaled model. The trends in terms of downstream distance and yaw misalignments (not shown here) are however extremely similar.

- The effects of blockage are very limited in the large wind tunnel of the Politecnico di Milano, with differences in power of about $1.5 \%$ and negligible effects on other metrics. 
- The effects of rotational augmentation, unmatched inboard circulation and nacelle size are clearly visible in the inner near wake region. However, they decay quickly with downstream distance, and are typically small enough not to alter the qualitative shape of the speed deficit, turbulence intensity and shear stresses distributions in this region of the wake.

- The lower ILS of the flow generated in the wind tunnel at the scale of the G1 has very modest effects on mean wake metrics, although it causes a reduced meandering.

In summary, it appears that the G1 scaled turbine faithfully represents not only the far wake behavior, but also produces a very realistic near wake. This is obtained by a design of the experimental setup that matches the turbulent inflow, the rotor vortex shedding, the geometry and strength of the helical tip vortices and the strength and shape of the speed deficit, which are all the main physical effects dictating the near-wake evolution. The mismatches that are present in the near-wake inner core (due to a different swirl, inboard circulation, rotational augmentation and a different geometry of the nacelle) do leave a visible mark, but overall do not seem to significantly alter the behavior of the wake, as expected. The larger size of the tower leaves a more visible trace further downstream, because it affects the wake recovery by generating a local extra turbulence intensity, in turn altering shear below hub height.

Overall, the realism of both the near and far wake justify the use of the TUM G1 (and similarly designed) scaled turbine for the study of wake physics and applications in wind farm control and wake mixing.

The present experimental setup can be further improved, for an even increased realism and expanded capabilities. Regarding the inflow, several facilities have been recently designed or upgraded to generate unstable boundary layers (Chamorro and Porté-Agel, 2010), tornadoes and downbursts (WindEEE, 2020), or for the active generation of turbulent flows (Kröger et al., 2018). Regarding the models, a more realistic geometry and size of the nacelle and tower can be achieved at the price of a further miniaturization. Aeroelastic effects can be included by using ad hoc scaling laws (Canet et al., 2020) to design flexible model rotor blades (Bottasso et al., 2014a; Campagnolo et al., 2014). Advances in 3D printing and component miniaturization will certainly lead to advancements in the design of ever more sophisticated and instrumented models. Regarding measurement technology, a more detailed characterization of salient features of the flow can be obtained by PIV or lidars, for example in support of the study of dynamic stall, vortex and stall-induced vibrations.

Although advancements in the testing of scaled wind turbines come with significant design, manufacturing, measurement and operational challenges, wind tunnel testing remains an extremely useful source of information for scientific discovery, the validation of numerical models and the testing of new ideas. A quantification of the realism of such scaled models is therefore a necessary step in the acceptance of the results that they generate. 
https://doi.org/10.5194/wes-2020-115

Preprint. Discussion started: 10 November 2020

(c) Author(s) 2020. CC BY 4.0 License.

(c) (i)

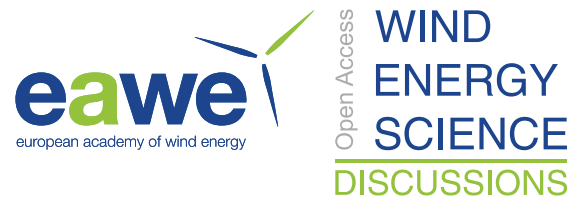

Author contributions. CW performed the simulations and analyzed the results; CLB devised the original idea of this research, performed the scaling analysis and supervised the work; FC was responsible for the wind tunnel experiments and the analysis of the measurements, and co-supervised the work; HC designed the full-scale turbine models; DB validated the full-scale turbine models with BEM and CFD

510 codes. CW and CLB wrote the manuscript. All authors provided important input to this research work through discussions, feedback and by improving the manuscript.

Competing interests. The authors declare that they have no conflict of interest.

Acknowledgements. The authors express their appreciation to the Leibniz Supercomputing Centre (LRZ) for providing access and computing time on the SuperMUC-NG System.

515 Financial support. This work has been supported by the CL-WINDCON project, which received funding from the European Union Horizon 2020 research and innovation program under grant agreement No. 727477. 
https://doi.org/10.5194/wes-2020-115

Preprint. Discussion started: 10 November 2020

(C) Author(s) 2020. CC BY 4.0 License.

\section{References}

Abkara, M., and Porté-Agel, F.: Influence of atmospheric stability on wind-turbine wakes: A large-eddy simulation study, Physics of Fluids, 27, 035104 https://doi.org/10.1063/1.4913695, 2015.

Bak, C., Zahle, F., Bitsche, R., Kim, T., Yde, A., Henriksen, L. C., Natarajan, A. and Hansen, M.: Description of the DTU 10 MW reference wind turbine, DTU Wind Energy Report-I-0092 5, 2013.

Bartl, J., and Sætran, L. R: Experimental testing of axial induction based control strategies for wake control and wind farm optimization, J. Phys. Conf. Ser., 753.3, https://doi.org/10.1088/1742-6596/753/3/032035, 2016.

Bastankhah, M. and Porté-Agel, F.: Experimental and theoretical study of wind turbine wakes in yawed conditions, J. Fluid Mech., 806, 506-541, https://doi.org/10.1017/jfm.2016.595, 2016.

Bisplinghoff, R. L. and Ashley, H.: Principles of Aeroelasticity, Dover Publications, Mineola, New York, USA, 2002.

Bottasso, C. L. and Campagnolo, F.: Handbook of Wind Energy Aerodynamics, chap. Wind Tunnel Testing of Wind Turbines and Farms, Springer, 2020.

Bottasso, C. L., Campagnolo, F. and Petrović, V.: Wind tunnel testing of scaled wind turbine models: Beyond aerodynamics, J. of Wind Eng. Indust. Aerodyn., 127, 11-28, https://doi.org/10.1016/j.jweia.2014.01.009, 2014.

Bottasso, C. L., Cacciola, S. and Iriarte, X.: Calibration of wind turbine lifting line models from rotor loads, Wind Eng. Ind. Aerod., 124, 29-45, https://doi.org/10.1016/j.jweia.2013.11.003, 2014.

Burton, T., Jenkins, N., Sharpe, D. and Bossanyi, E.: Wind energy handbook, John Wiley \& Sons, West Sussex, UK, 2011.

Campagnolo, F., Bottasso, C. L., and Bettini, P.: Design, manufacturing and characterization of aero- elastically scaled wind turbine blades for testing active and passive load alleviation techniques within an ABL wind tunnel, J. Phys. Conf. Ser., 524 012061, https://doi.org/10.1088/1742- 6596/524/1/012061, 2014.

Campagnolo, F., Petrović, V., Schreiber, J., Nanos, E. M., Croce, A. and Bottasso, C. L.: Wind tunnel testing of a closed-loop wake deflection controller for wind farm power maximization, J. Phys. Conf. Ser., 753.3, https://doi.org/10.1088/1742-6596/753/3/032006, 2016.

Campagnolo, F., Weber, R., Schreiber, J. and Bottasso, C. L.: Wind tunnel testing of wake steering with dynamic wind direction changes, Wind Energy Sci. Discuss., https://doi.org/10.5194/wes-2020-70, 2020 .

Canet, H., Bortolotti, P., and Bottasso, C. L.: On the scaling of wind turbine rotors, Wind Energy Sci. Discuss., pp. 1-35, 2020.

Chamorro, L. P. and Porté-Agel, F.: A wind-tunnel investigation of wind-turbine wakes: boundary-layer turbulence effects, Bound.-Layer Meteorol., 132.1, 129-149, https://doi.org/10.1007/s10546-009-9380-8, 2009.

Chamorro, L. P. and Porté-Agel, F.: Effects of thermal stability and incoming boundary-layer flow characteristics on wind-turbine wakes: a wind-tunnel study, Bound.-Layer Meteorol., 136.3, 515-533, https://doi.org/10.1007/s10546-010-9512-1, 2010.

Chamorro, L. P., Arndt, R. E. A. and Sotiropoulos, F.: Reynolds number dependence of turbulence statistics in the wake of wind turbines, Wind Energy, 15.5, 733-742, https://doi.org/10.1002/we.501, 2012.

Chen, T. Y. and Liou, L. R.: Blockage corrections in wind tunnel tests of small horizontal-axis wind turbines, Exp. Therm. Fluid Sci., 35.3, 565-569, https://doi.org/10.1016/j.expthermflusci.2010.12.005, 2011.

550 Churchfield, M. J., Schreck, S., Martınez-Tossas, L. A., Meneveau, C., and Spalart, P. R.: An advanced actuator line method for wind energy applications and beyond, in: 35th Wind Energy Symposium, p. 1998, 2017.

Dowler J. L. and Schmitz, S.: A solution-based stall delay model for horizontal-axis wind turbines, Wind Energy, 18, 1793-1813, https://doi.org/10.1002/we.1791, 2015. 
https://doi.org/10.5194/wes-2020-115

Preprint. Discussion started: 10 November 2020

(C) Author(s) 2020. CC BY 4.0 License.

(c) (i)

España, G., Aubrun, S., Loyer, S., and Devinant, P.: Spatial study of the wake meandering using modelled wind turbines in a wind tunnel, Wind Energy, 14, 923-937, https://doi.org/10.1002/we.515, 2011.

Fleming, P. A., Pieter, M. O., Lee, S., Wingerden, J., Johnson, K., Churchfield, M., Michalakes, J., Spalart, P. and Moriarty, P.: Evaluating techniques for redirecting turbine wakes using SOWFA, Renew. Energy, 70, 211-218, https://doi.org/10.1016/j.renene.2014.02.015, 2014.

IEC 61400-1 Wind turbine generator systems - Part 1: Safety requirements. 2nd edition. Geneva, Switzerland: International Electrotechnical Commission, 1999.

560 IEC 61400-1 Wind turbines - Part 1: Design requirements. 3rd edition. Geneva, Switzerland: International Electrotechnical Commission, August 2005.

Jasak, H., Weller, H., and Gosman, A.: High resolution NVD differencing scheme for arbitrarily unstructured meshes, Int. J. Numer. Methods Fluids, 31, 431-449, https://doi.org/10.1002/(SICI)1097-0363(19990930)31:2<431::AID-FLD884>3.0.CO;2-T, 1999.

Jasak, H.: OpenFOAM: open source CFD in research and industry, Int. J. Nav. Arch. Ocean, 1.2, 89-94, https://doi.org/10.2478/IJNAOE2013-0011, 2009.

Jasak, H. and Rigler, D.: Finite volume immersed boundary method for turbulent flow simulations, in: 9th OpenFOAM Workshop, 2014.

Jonkman, B.J.: TurbSim User's Guide: Version 1.50, Technical Report NREL/TP-500-46198, September 2009.

Jonkman, J. and Jonkman, B.J.: FAST 8, https://nwtc.nrel.gov/FAST8, 2018.

Kelly, M.: From standard wind measurements to spectral characterization: turbulence length scale and distribution, Wind Energ. Sci., 3, 533-543, https://doi.org/10.5194/wes-3-533-2018, 2018.

Kröger, L., Frederik, J., van Wingerden, J.-W., Peinke, J., and Hölling, M.: Generation of user defined turbulent inflow conditions by an active grid for validation experiments, J. Phys. Conf. Ser., 1037(5), 2018.

Fleming, P., Annoni, J., Churchfield, M., Martınez-Tossas, L. A., Gruchalla, K., Lawson, M., and Moriarty, P.: A simulation study demonstrating the importance of large-scale trailing vortices in wake steering, Wind Energy Science, 3, 243-255, https://doi.org/10.5194/wes-3243-2018, 2018.

Lissaman, P. $\quad$ B. $\quad$ S.: Low-Reynolds-number airfoils, Annual Review of Fluid Mechanics, 15(1), https://doi.org/10.1146/annurev.fl.15.010183.001255, 1983.

Lyon, C. and Selig, M. S.: Summary of low speed airfoil data, Soartech Publications, Virginia, USA, 1997.

Martínez-Tossas, L. A., Churchfield, M. J. and Leonardi, S.: Large eddy simulations of the flow past wind turbines: actuator line and disk modeling, Wind Energy, 18.6, 1047-1060, https://doi.org/10.1002/we.1747, 2015.

Meinhart, C. D., Wereley, S. T., and Santiago, J. G.: PIV measurements of a microchannel flow, Exp. Fluids., 27.5, 414-419, https://doi.org/10.1007/s003480050366, 1999.

Mittal, R. and Iaccarino, G.: Immersed boundary methods, Annu. Rev. Fluid Mech, 37, 239-261, https://doi.org/10.1146/annurev.fluid.37.061903.175743, 2005.

Okulov, V. L. and Sørensen, J. N.: Stability of helical tip vortices in a rotor far wake, J. Fluid Mech. 576, 1-25, https://doi.org/10.1017/S0022112006004228, 2007.

Pitt, D. M., and Peters, D. A.: Theoretical prediction of dynamic-inflow derivatives, Vertica, 5.1, 21-34, https://doi.org/20.500.11881/1796, 1981.

Shipley, D. E., Miller, M. S., and Robinson, M. C.: Dynamic stall occurrence on a horizontal axis wind turbine blade, in: CONF-950116-8, National Renewable Energy Lab., Golden, CO, US, 1995. 
https://doi.org/10.5194/wes-2020-115

Preprint. Discussion started: 10 November 2020

(C) Author(s) 2020. CC BY 4.0 License.

(c) (i)

Smagorinsky, J.: General circulation experiments with the primitive equations: I. The basic experiment, Monthly weather rev., 91.3, 99-164, https://doi.org/10.1175/1520-0493(1963)091<0099:GCEWTP>2.3.CO;2, 1963.

Snel, H., Houwink, R. and Bosschers, J.: Sectional prediction of lift coefficients on rotating wind turbine blades in stall. Netherlands Energy Research Foundation, Petten, Netherlands, 1994.

Sørensen, J. N.: Instability of helical tip vortices in rotor wakes, J. Fluid Mech., 682, 1-4, https://doi.org/10.1017/jfm.2011.277, 2011.

Tian, W., Ozbay, A. and Hu, H.: An experimental investigation on the wake interferences among wind turbines sited in aligned and staggered wind farms, Wind Energy, 21.2, 100-114, https://doi.org/10.1002/we.2147, 2018.

Troldborg, N., Sørensen, J. N. and Mikkelsen, R.: Actuator line simulation of wake of wind turbine operating in turbulent inflow, J. Phys. Conf. Ser., 75.1, https://doi.org/10.1088/1742-6596/75/1/012063, 2007.

van der Laan, M. P., and Sørensen, N. N.: Why the Coriolis force turns a wind farm wake to the right in the Northern Hemisphere, J. Phys.: Conf. Ser. 753 032031, https://doi.org/10.1088/1742-6596/753/3/032031, 2016.

van Dooren, M. F., Campagnolo, F., Sjöholm, M., Angelou, N. and Mikkelsen, T.: Demonstration and uncertainty analysis of synchronised scanning lidar measurements of 2-D velocity fields in a boundary-layer wind tunnel, Wind Energy Science, 2, 329-341, https://doi.org/10.5194/wes-2-329-2017, 2017.

Wang, C., Wang, J., Campagnolo, F., Carraón, D. B. and Bottasso, C. L.: Validation of large-eddy simulation of scaled waked wind turbines in different yaw misalignment conditions, J. Phys. Conf. Ser., 1037 062007, https://doi.org/10.1088/1742-6596/1037/6/062007, 2018.

Wang, J., Wang, C., Campagnolo, F. and Bottasso, C. L.: Wake behavior and control: comparison of LES simulations and wind tunnel measurements, Wind Energy Sci., 4.1, 71-88, https://doi.org/10.5194/wes-4-71-2019, 2019.

Wang, C., Campagnolo, F. and Bottasso, C. L.: Identification of airfoil polars from uncertain experimental measurements, Wind Energy Sci., accepted, to appear, 2020.

Wang, C., Muñoz-Simón, A. Deskos, G., Laizet, S., Palacios, R., Campagnolo, F. and Bottasso, C. L.: Code-to-code-to-experiment validation of LES-ALM wind farm simulators, J. Phys. Conf. Ser. 1618 062041, https://doi.org/10.1088/1742-6596/1618/6/062041, 2020.

Wang, C., Campagnolo, F., Sharma, A. and Bottasso, C. L.: Effects of dynamic induction control on power and loads, by LES-ALM simulations and wind tunnel experiments, J. of Phys.: Conf. Ser. 1618 022036, https://doi.org/10.1088/1742-6596/1618/2/022036, 2020.

Whale, J., Papadopoulos, K. H., Anderson, C. G., Helmis, C. G., and Skyner, D. J.: A study of the near wake structure of a wind turbine comparing measurements from laboratory and full-scale experiments, Solar Energy, 56.6, https://doi.org/10.1016/0038-092X(96)00019-9, 1996.

WindEEE https://www.eng.uwo.ca/windeee/, last accessed: 13 October 2020.

$\mathrm{Wu}$, Y. and Porté-Agel, F.: Large-eddy simulation of wind-turbine wakes: evaluation of turbine parametrisations, Boundary-layer Meteorol., 138.3, 345-366, https://doi.org/10.1007/s10546-010-9569-x, 2011.

Zhan, L., Letizia, S., and Iungo, G. V.: LiDAR measurements for an onshore wind farm: Wake variability for different incoming wind speeds and atmospheric stability regimes, Wind Energy, 23.3, 501-527, https://doi.org/doi.org/10.1002/we.2430, 2020. 